\title{
Time-resolved spectroscopy of $\mathrm{BD}+46^{\circ} 442$ : Gas streams and jet

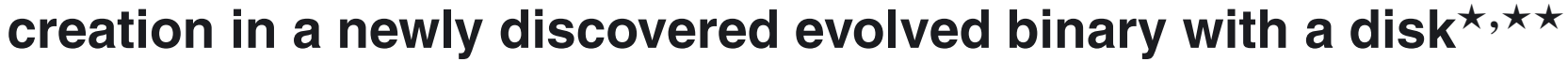

\author{
N. Gorlova ${ }^{1}$, H. Van Winckel ${ }^{1}$, C. Gielen ${ }^{1}$, G. Raskin ${ }^{1}$, S. Prins ${ }^{1}$, W. Pessemier ${ }^{1}$, C. Waelkens ${ }^{1}$, Y. Frémat ${ }^{2}$, \\ H. Hensberge ${ }^{2}$, L. Dumortier ${ }^{2}$, A. Jorissen ${ }^{3}$, and S. Van Eck ${ }^{3}$ \\ ${ }^{1}$ Instituut voor Sterrenkunde, Katholieke Universiteit Leuven, Celestijnenlaan 200D, 3001 Leuven, Belgium \\ e-mail: nadya@ster.kuleuven.be \\ 2 Royal Observatory of Belgium, 3 avenue Circulaire, 1180 Brussels, Belgium \\ 3 Institut d'Astronomie et d’Astrophysique, Université Libre de Bruxelles, CP 226, Boulevard du Triomphe, 1050 Bruxelles, Belgium
}

Received 23 December 2011 / Accepted 5 April 2012

\section{ABSTRACT}

\begin{abstract}
Context. Previous studies have shown that many post-asymptotic giant branch (AGB) stars with dusty disks are associated with single-lined binary stars. The inferred orbital separations are too small to accommodate a fully grown AGB star, hence these systems represent a new evolutionary channel that bypasses a full AGB evolution.

Aims. We wish to verify the binarity hypothesis for a larger sample establish the nature of the companions, and probe the disk structure and eventually the disk formation mechanisms in binary stars. To achieve these aims, we started a high-resolution spectral monitoring of $\sim 40$ field giants whose binarity had been suspected based on either a light curve, an infrared excess, or a peculiar chemical composition.

Methods. Starting from the spring of 2009, we monitored the programme stars with the fibre echelle spectrometer HERMES. We measure their radial velocities (RVs) with a precision of $\sim 0.2 \mathrm{~km} \mathrm{~s}^{-1}$, perform detailed photospheric abundance analyses, and analyse the time-resolved high-resolution spectra to search for line-profile variability.

Results. Here we report on the discovery of periodic RV variations in $\mathrm{BD}+46^{\circ} 442$, a high Galactic latitude $\mathrm{F}$ giant with a disk. We infer that the variations are caused by the motion around a faint companion, and deduce the orbital parameters $P_{\text {orb }}=140.77 \pm 0.02^{\mathrm{d}}, e=$ $0.083 \pm 0.002$, and $a \sin i=0.31 \mathrm{AU}$. We find that it is a moderately metal-poor star $([\mathrm{M} / \mathrm{H}]=-0.7)$ without a strong depletion pattern in its photospheric abundances. Interestingly, many lines indeed show periodic changes with the orbital phase: H $\alpha$ switches between a double-peak emission line and a P Cyg-like profile, while strong metal lines appear to be split at the maximum redshift. Similar effects are likely visible in the spectra of other post-AGB binaries, but their regularity is not always apparent owing to sporadic observations. We propose that these features result from an ongoing mass transfer from the evolved giant to the companion. In particular, the blueshifted absorption in $\mathrm{H} \alpha$, which occurs only at superior conjunction, may result from a jet originating in the accretion disk around the companion and that is seen in absorption towards the luminous primary.
\end{abstract}

Key words. stars: individual: BD $+46^{\circ} 442$ - binaries: spectroscopic - circumstellar matter - stars: AGB and post-AGB ISM: jets and outflows - stars: abundances

\section{Introduction}

Van Aarle et al. (2011) investigated the spectral energy distribution (SED) of optically bright candidate post-asymptotic giant branch (post-AGB) stars in the Large Magellanic Cloud (LMC). Surprisingly, they found that in about half of their candidates, the SED indicates that the circumstellar material, which is a relic of the AGB mass loss, is not in the form an expanding envelope, but rather a stable, likely Keplerian, disk.

In our own Galaxy, post-AGB stars with disks are also common (e.g. De Ruyter et al. 2006), but the statistics of Galactic post-AGB stars are less complete than in the LMC, and the lack of well-constrained distances (hence luminosities) makes the

\footnotetext{
* Based on observations made with the Mercator Telescope, operated on the island of La Palma by the Flemish Community, at the Spanish Observatorio del Roque de los Muchachos of the Instituto de Astrofísica de Canarias.

$\star \star$ Appendix $\mathrm{A}$ is available in electronic form at http://www. aanda.org
}

interpretation of these numbers more difficult. Galactic sources, however, have an obvious advantage of being closer and easier to study.

The leading explanation of the channelling of matter into either outflows or disks, is that disks form only around binaries. The observational support for this is that indeed, many disk sources in our Galaxy have been found to be single-lined binaries (e.g. Van Winckel et al. 2009, and references therein). Disk formation presumably occurs by means of an interaction with the companion when the more massive component becomes either a red giant (RG) or an AGB star (Van Winckel 2003). The process itself is poorly understood. Livio \& Soker (1988) and Sandquist et al. (1998), among others, showed that a circumbinary disk could be a remnant of a common envelope created when the Roche lobe of an AGB star engulfs a companion. Other scenarios have been proposed, such as that of Mastrodemos \& Morris (1999) where a disk-like structure is created in a windaccretion scenario or perhaps as the result of the AGB wind and the accretion jet interaction (Akashi \& Soker 2008). 
The impact of the disk on the system cannot be underestimated. Many of the Galactic and even LMC post-AGB disk objects have a peculiar chemical photospheric composition that resembles the gas phase of the interstellar medium (ISM): elements of higher condensation temperature, such as iron, are less abundant than volatiles (e.g. Giridhar et al. 2005; Maas et al. 2005; Gielen et al. 2009; Sumangala Rao et al. 2012). This is believed to be the result of pollution by a disk, which may have already dissipated in some systems and therefore has no footprint in the SED. In the disk, refractory elements nucleate into dust grains, which are segregated from the gas, and the depleted gas falls back onto the star (Waters et al. 1992). On the one hand, this effect helps us to identify either current or former disk systems, but on the other hand masks the original metallicity of the stars and the eventual products of the AGB dredge-ups, complicating the identification of the evolutionary stage.

At a later evolutionary stage, as proposed by Waelkens et al. (1996) and Jorissen (1999), a disk system may turn into a barium star, when the current post-AGB primary evolves into a white dwarf (WD) and a supposedly main-sequence (MS) companion into a red giant. Barium stars are binaries where a red giant bears signs of contamination by the nuclear-synthetic products (such as barium) by the former AGB companion (now a WD). By adopting the formalism of Lubow \& Artymowicz (1996), Dermine et al. (2012) explored the binary-disk interaction regime relevant to barium stars and showed that circumbinary disks may play an important role in pumping the eccentricities of barium stars to the observed values.

The Galactic objects with known orbits cannot have evolved on single-star evolutionary tracks: a typical AGB star has a radius $\sim 1$ AU, while many post-AGB binaries with a disk have smaller separations, which raises a possibility that these systems had already started interacting at the red giant stage. Some of the objects may never have gone through the AGB stage, unless the interaction resulted in an orbital shrinkage. The observed periods indicate that the full AGB evolution must have been curtailed, an idea that is corroborated by the finding that the majority of disk sources have oxygen-rich photospheres and disks dominated by crystalline silicates (Gielen et al. 2008, 2011). Another uncertainty in the evolution of these systems is whether these stars become planetary nebulae (PNe), since the circumstellar material locked in a disk. While binarity helps us to explain bipolar $\mathrm{PNe}$, the periods of binary stars in PNe are at least two orders of magnitude shorter than in the disk post-AGB systems (De Marco 2009).

The theories of disk formation that have been developed are similarly unable to predict the timescales for disk re-accretion and dissipation. Some insight can be gained by gathering information on the innermost regions of the disk from optical and near-IR spectroscopy. Previous spectroscopic studies of postAGB stars have mainly explored stellar radial velocities (RVs) and the basic properties of both the photosphere and the disk. Only briefly did they consider the signs of activity at the starto-disk interface, such as either emission in $\mathrm{H} \alpha$ or circumstellar absorption in the CaII H\&K and NaI D lines, and the common interpretation has been that these features trace a subsiding AGB wind. The notion was that after a circumbinary disk is formed, the system enters passive evolution as a detached binary. No success has been achieved in identifying gas re-accretion (with only one tentative detection of the winnowing gas by Hinkle et al. 2007). In none of the systems the companion has been detected so far. To explore the details of the disk-star interaction, a systematic time-resolved investigation of the disk and chemically peculiar post-AGB systems is therefore highly desirable.

A literature compilation of Galactic post-AGBs was assembled in the Torun catalogue (Szczerba et al. 2007) ${ }^{1}$ and consists of $\sim 400$ objects. In this catalogue, 51 sources have been identified as disk sources in De Ruyter et al. (2006), and this number has continued to increase. About two dozen post-AGB stars in De Ruyter et al.'s list have been identified as single-lined spectroscopic binaries with periods from 115 to 2600 days and orbital separations from 0.1 to 3.6 AU. The latest addition can be found in Van Winckel et al. (2009), who photometrically and spectroscopically monitored a selection of low-amplitude pulsators from the southern hemisphere. All low-amplitude pulsators are binaries, but the suspected binary nature of the remaining $60 \%$ of the stars in De Ruyter et al's list remains to be established. This is an observational challenge, because many objects display large amplitude pulsations that prevent a straightforward interpretation of radial velocity variations (e.g. Maas et al. 2002).

In the spring of 2009, we started a high-resolution spectroscopic monitoring programme of 34 Galactic post-AGB candidates with disks and a few objects without an IR excess, that are observable from the northern hemisphere, using a newly built optical echelle spectrograph HERMES (Raskin et al. 2011). These objects were required to satisfy at least two of the following criteria: an IR excess characteristic of a disk, an RV Tau-like variability, a variability indicative of a possible obscuration by a disk, a low surface gravity combined with a location away from the Galactic plane, and a deficiency in the refractory elements. In this paper, we report on one of the first new short-period binaries that we uncovered after three years of observations, BD+46 442 (SAO 37487, IRAS 01427+4633), which is a poorly studied $V=9.5$ high Galactic latitude $\mathrm{F}$ giant $^{2}\left(b=15^{\circ}\right)$, not known to be variable in RV or photometry. It was selected for inclusion in our survey because of its strong infrared excess, which is characteristic of disk objects, as originally measured by the IRAS satellite. The paper is organized as follows: after a description of the data (Sect. 2), we present our results of the abundance analyses of BD $+46^{\circ} 442$ (Sect. 3), then model its SED (Sect. 4) and radial velocity (RV) curve (Sect. 5), and examine the behaviour of both the hydrogen and some metal lines (Sect. 6). In our discussion in Sect. 7, we compare the discovered periodic spectral variations with those in both other post-AGB systems and interacting binaries, and discuss theories capable of explaining this behaviour.

\section{Observations and data reduction}

Each target in our ongoing post-AGB survey is observed about twice per month during the visibility season. This programme is part of a much broader effort to study binary interaction processes in evolved stars (Van Winckel et al. 2010). The main goal is to measure RVs to detect and characterize the orbital motion. For brighter targets, additional studies are performed, such as the determination of abundances, rotation velocities, and circumstellar features. The spectra are obtained with the fibre-fed echelle spectrograph HERMES, attached to the Flemish $1.2 \mathrm{~m}$ telescope Mercator on La Palma, Canary Islands. The spectrograph is optimized for high resolution, stability, and a broad wavelength coverage. This is achieved primarily by implementing an

\footnotetext{
1 http://www . ncac. torun. pl/postagb2

2 When referring to $\mathrm{BD}+46^{\circ} 442$-like systems, the term "giant" is used to indicate a low surface-gravity, extended, post-MS object. The exact luminosity class and the evolutionary stage will only be able to be established when the parallax information becomes available.
} 
image slicer, an anti-fringe CCD coating, and a thermal enclosure (Raskin et al. 2011).

Sixty spectra of BD $+46^{\circ} 442$ were obtained during three seasons of observations in 2009-2012. We used a fibre configuration that provides a resolution $R=80000-90000$ over the wavelength range $\Delta \lambda=3800-9000 \AA$. The exposure times varied across the range 500-1800 s, while the signal-to-noise ratio (S/N) was 20-90 (as measured near $6500 \AA$ ). Nightly calibrations consisted of two sets of biases, flats, and the Th-Ne-Ar arc spectra obtained in both the evening and the morning. To reduce a particular object frame, the nearest set of calibration data was used. At least one International Astronomical Union radial velocity standard was observed nightly and occasionally a series of arcs, to monitor the fluctuations in the zero-point of the wavelength calibration. These tests demonstrated that the typical uncertainty in the absolute value of the RV is $\sim 0.2 \mathrm{~km} \mathrm{~s}^{-1}$ and is mainly caused by the pressure fluctuations in the instrument room (Raskin et al. 2011). The data reduction was performed with a dedicated Python-based pipeline that produces extracted, cosmic-ray cleaned, wavelength calibrated, and order-merged spectra.

\section{Atmospheric analysis}

\subsection{Atomic data and model atmospheres}

Our atmospheric analysis of $\mathrm{BD}+46^{\circ} 442$ was based on a comparison with synthetic profiles of hydrogen lines and the equivalent-width (EW) measurement of metal lines. The latter were obtained using the DECH20 code by Galazutdinov $(1992)^{3}$, which provides a convenient interface for the continuum normalization, and the EW measurement by means of either a Gaussian fit or a direct integration. Line identification was carried out using the following on-line resources: the Spectroweb stellar atlas of Lobel $(2008,2011)^{4}$ for $\lambda<6800 \AA$, and the Solar atlas of Delbouille et al. $(1988)^{5}$ in combination with the atomic line database of Van $\mathrm{Hoof}^{6}$ for the longer wavelengths.

The line list used for the abundance determination was provided by Kovtyukh (2010, priv. comm.) and was mainly based on the version published in Kovtyukh \& Andrievsky (1999). We also followed the same procedure as these authors to determine the surface gravity $(\log g)$ and the micro-turbulent velocity $\left(V_{\text {tur }}\right)$. Our choice was dictated by the yellow supergiants studied by Kovtyukh \& Andrievsky (1999) spectroscopically being very similar to post-AGB stars. To convert EWs to abundances, we used the local thermodynamical equilibrium (LTE) radiative transfer code MOOG developed by Sneden ${ }^{7}$ with the ATLAS9 model atmospheres, which incorporate the updated opacity distribution functions of Castelli \& Kurucz (2003) ${ }^{8}$.

Kovtyukh \& Andrievsky (1999) obtained the bulk of their oscillator strengths $(\log g f$-s) by adjusting the laboratory values to ensure that they could reproduce the solar abundances of Grevesse et al. (1996) from the EWs measured in the solar spectrum of Kurucz et al. (1984). They used the canonical solar model of $5777 / 4.438 / 1.0\left(T_{\text {eff }} / \log g / V_{\text {tur }}\right)$ derived from the set of the original ATLAS9 models of Kurucz (1992) and his

\footnotetext{
${ }^{3}$ http://www.gazinur.com/DECH-software.html

4 http://spectra.freeshell.org/spectroweb.html

5 http://ljr.bagn.obs-mip.fr/observing/spectrum.html

${ }^{6}$ http://www.pa.uky.edu/ peter/atomic/

7 http://www.as.utexas.edu/ chris/moog.html

${ }^{8}$ http://wwwuser.oat.ts.astro.it/castelli/grids.html
}

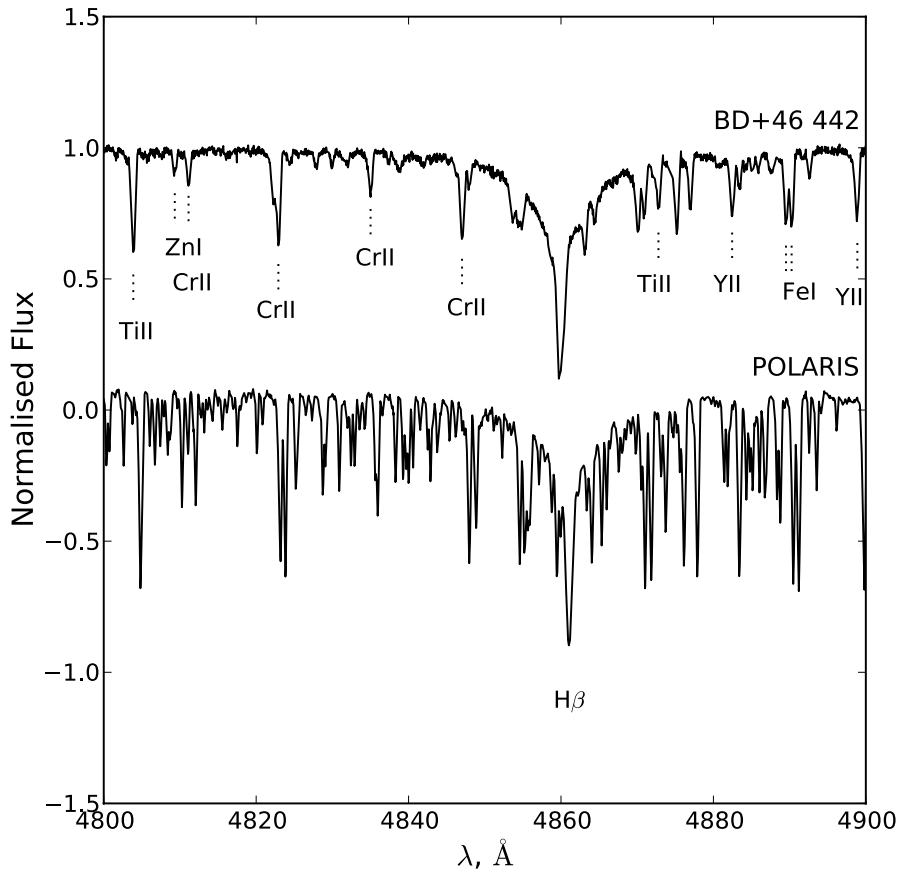

Fig. 1. Comparison of the spectrum of $\mathrm{BD}+46^{\circ} 442$ obtained on 17 August 2009 with the HERMES spectrum of Polaris. While $\mathrm{H} \beta$ is similar in both stars, indicating similar temperature and gravity, metal lines are noticeably weaker (and broader) in $\mathrm{BD}+46^{\circ} 442$, which is indicative of a sub-solar metallicity.

WIDTH9 LTE code ${ }^{9}$. We found that this combination of older atmospheric models and code provided for a given element abundances that were systematically slightly different than those derived using our combination of atmospheric models and code, both for the Sun and BD $+46^{\circ} 442$. Therefore, when giving abundances of $\mathrm{BD}+46^{\circ} 442$ relative to the $\operatorname{Sun}([\mathrm{X} / \mathrm{H}])$, we introduced small corrections (up to $\pm 0.08 \mathrm{dex}$ ) into the solar abundances of Kovtyukh \& Andrievsky (1999) to compensate for this difference.

\subsection{Photospheric parameters}

Since there is no information about either the reddening or the photometric variability in $\mathrm{BD}+46^{\circ} 442$, we decided to derive all photospheric parameters spectroscopically. To achieve this, we analysed a $S / N \sim 130$ spectrum obtained by averaging the highest quality spectra numbered 3 and 4 from the same night (Fig. 1). The visual examination of all the spectra did not reveal any obvious variations in the effective temperature $\left(T_{\text {eff }}\right)$. Variations in the cores of the strongest lines have been detected, which we attribute to the circumstellar matter (Sect. 6), although these lines were not used in the EW analysis (we only used lines with $E W s \leq 170 \AA$ ). This single spectrum, therefore, should provide a good representation of the basic photospheric parameters of our star.

We first confirmed the spectral type F from Simbad by comparing our spectrum to the spectral libraries of Gray ${ }^{10}$ and the UVES spectrograph at the Very Large Telescope (Bagnulo et al. $2003)^{11}$. We then computed the iron abundance from our EWs,

\footnotetext{
9 http://wwwuser.oat.ts.astro.it/castelli/sources/ width9.html

${ }^{10}$ http://ned.ipac. caltech.edu/level5/Gray/

Gray_contents.html

11 http://www.sc.eso.org/santiago/uvespop/
} 
by adopting the range of model parameters expected for an F I-III star of $T_{\text {eff }}=6200-8000 \mathrm{~K}, \log g=0.5-4.0$, and $V_{\text {tur }}=0-12.0 \mathrm{~km} \mathrm{~s}^{-1}$; we used the ATLAS9 models with solar metallicity $([\mathrm{M} / \mathrm{H}]=0)$ and later $[\mathrm{M} / \mathrm{H}]=-0.5$ to match the subsolar metallicity obtained in the final iteration. To perform an excitation analysis, we plotted the iron abundances against the EW and the excitation potential of the lower level $(\chi)$, separately for the Fe I and Fe II lines, to examine any possible trends. Correct (LTE) atmospheric parameters are obtained when the EWs of all the lines correspond to the same value of the abundance. Trends with the EW can be removed by tuning $V_{\text {tur }}$, while agreement between the average abundances derived from the $\mathrm{Fe} I$ and Fe II lines (the ionization balance) implies that the value of $\log g$ is correct. Iron lines are usually used in this method because they are the most numerous, but the same values of $V_{\text {tur }}$ and $\log g$ should in principle hold for all the elements.

At sub-solar metallicities and gravities, however, neutral species become underpopulated relative to the LTE case, owing to the ultraviolet over-ionization and the lower rate of collisions, resulting in the positive corrections that need to be added to the LTE abundances (Rentzsch-Holm 1996; Bergemann 2011; Mashonkina et al. 2011). Observationally, this is manifested in the case of the supergiants, where Fe I lines require smaller values of $V_{\text {tur }}$ and $\log g$ to bring their abundances into agreement with the more well-behaved Fe II lines (which is the dominant ionization state in F-G stars), as shown for example in Kovtyukh $\&$ Andrievsky (1999) for the classical Cepheid $\delta$ Cep and in Takeda et al. (2007) for some post-AGB stars. We found the same effect for $\mathrm{BD}+46^{\circ} 442$. Following recommendations in the literature, we used the Fe II lines to determine $V_{\text {tur }}$, while for the determination of $\log g$ we extrapolated abundances from the Fe I lines to $E W=0$, as weak lines are less susceptible to nonLTE effects. Following this procedure, we found a correlation between the adopted $T_{\text {eff }}$ and the derived $\log g /[\mathrm{Fe} / \mathrm{H}]$. To break this degeneracy, we turned to the Balmer and Paschen hydrogen lines.

Hydrogen lines have the advantage over the metal lines that to first order they do not depend on the metallicity. Furthermore, we can consider the shape of the extended wings in addition to the line depth when comparing to the synthetic profiles. We compared Balmer lines in $\mathrm{BD}+46^{\circ} 442$ to the synthetic profiles of Coelho et al. (2005) $)^{12}$ and Paschen lines to those of Munari \& Castelli (2000) ${ }^{13}$. The $\mathrm{H} \alpha$ line was not considered, as it shows a strong non-photospheric contribution in all phases. From the Paschen lines, we chose to consider only Pa14 and Pa17 as the least blended ones. We noticed that the Paschen break in the models is far deeper than in $\mathrm{BD}+46^{\circ} 442$ and the other supergiants that we studied; therefore, before making a comparison, we had to normalize the model and the observed profiles at the pseudo-continuum regions between the neighbouring lines.

In the parameter space considered here, model hydrogen lines strengthen both with higher $T_{\text {eff }}$ and lower $\log g$ (Fremat et al. 1996; Munari \& Castelli 2000). Fortunately, a stronger sensitivity of Paschen lines to gravity allowed us to exclude $\log g \geq 3.0$, and $T_{\text {eff }}>6500 \mathrm{~K}$ along with it. No Paschen models are available for $\log g<2$ for the range of temperatures considered here, but they would require $T_{\text {eff }}<6000 \mathrm{~K}$, which is too low to fit Balmer lines. We therefore adopted $T_{\text {eff }}=6250 \pm 250 \mathrm{~K}, \log g<3.0$ (Fig. 2), and returned to the iron

\footnotetext{
12 http://wWw.mpa-garching.mpg.de/PUBLICATIONS/DATA/ SYNTHSTELLIB/synthetic_stellar_spectra.html

${ }^{13}$ http://vizier.u-strasbg.fr/viz-bin/VizieR-4? - source=III/238
}
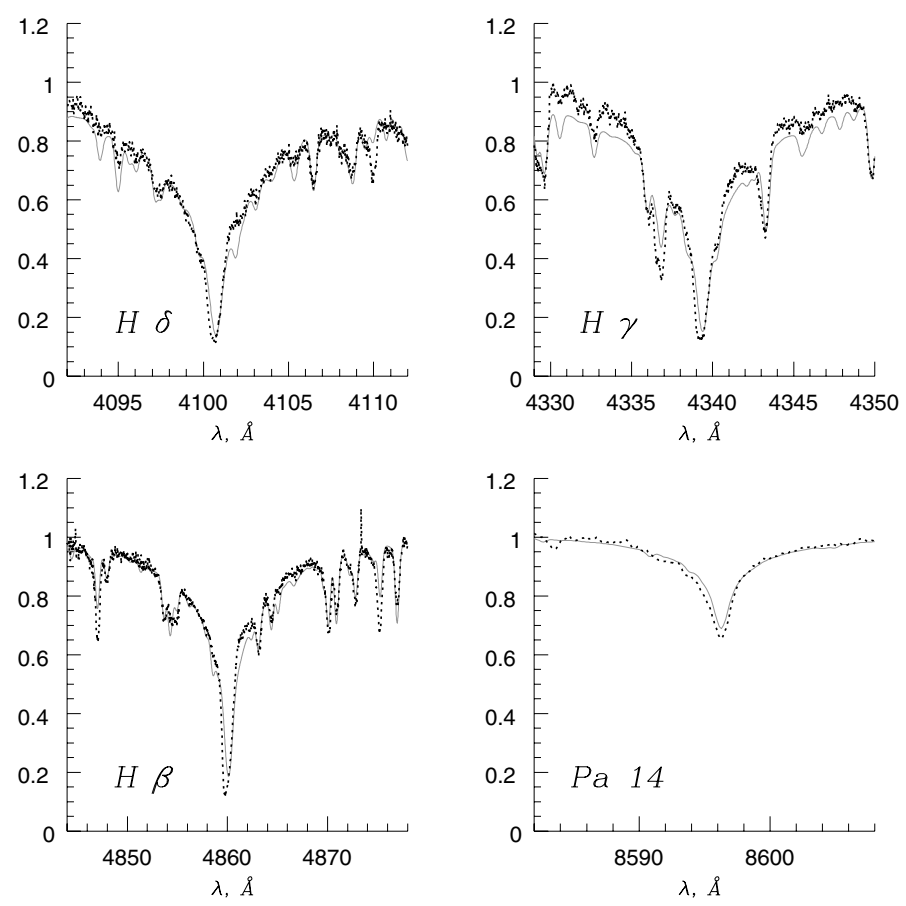

Fig. 2. Hydrogen lines in $\mathrm{BD}+46^{\circ} 442$ (black dots) compared to the most closely fitting of the available synthetic profiles (grey solid lines), that were computed with $T_{\text {eff }}=6250 \mathrm{~K}, \log g=2.0,[\mathrm{M} / \mathrm{H}]=-0.5$. To match the resolution of the model Paschen lines, the observed profiles were convolved to $R=20000$, while Balmer lines are shown at the native HERMES resolution of 85000 .

lines to place a tighter constraint on $\log g$. Our estimate is confirmed by a close match in terms of both the Paschen lines and the shape of $\mathrm{H} \beta$ wings between $\mathrm{BD}+46^{\circ} 442$ and Polaris (Fig. 1). Polaris Aa is a well-studied short-period classical Cepheid, for which e.g., Usenko et al. (2005) inferred $T_{\text {eff }}=6000 \pm 170 \mathrm{~K}$, $\log g=2.2 \pm 0.3$, and $[\mathrm{Fe} / \mathrm{H}]=+0.07$.

Finally, $T_{\text {eff }}$ is also frequently derived from the requirement that the iron abundance derived from the Fe I lines does not depend on $\chi$. Applying this criterion to $\mathrm{BD}+46^{\circ} 442$, we obtained $T_{\text {eff }} \approx 7500 \mathrm{~K}$, which is inconsistent with the hydrogen fits. As we have discussed above, the abundances derived from Fe I lines may be unreliable in our star because of the non-LTE effects. On the other hand, Fe II lines do not cover a large enough range of excitation potentials. We thus restricted ourselves to $T_{\text {eff }}=6000$, 6250 , and $6500 \mathrm{~K}$ and, by performing $\mathrm{EW}$ analyses of iron lines, deduced the combinations of $\log g / V_{\text {tur }}$ for these temperatures of 1.0/4.0, 1.5/4.0, and 2.0/4.0, with a precision of $0.5 \mathrm{dex}$ in $\log g$ (set by the model step size), and $1 \mathrm{~km} \mathrm{~s}^{-1}$ for $V_{\text {tur }}$. Our final bestfit model of 6250/1.5/4.0 is shown in Fig. 3. Linear fits to the $\mathrm{Fe}$ II and Fe I abundances plotted against EWs (and extrapolated to $E W=0$ for $\mathrm{Fe}$ I) gave $6.74 \pm 0.07$ and $6.76 \pm 0.06$ for the iron abundance, respectively. The other two best-fit models were used to estimate the model-dependent errors in the abundances.

\subsection{Abundances}

The abundances of all elements deduced from the individual lines using our best model atmosphere are shown in Fig. 4. The elements are arranged in order of increasing condensation temperature to verify the presence of the depletion pattern reported in the literature for many disk sources. The average abundance per element and the error budget are shown in Fig. 5 and in Table 1. Given possible non-LTE effects, we assigned a quality 
N. Gorlova et al.: A newly discovered evolved binary with a disk
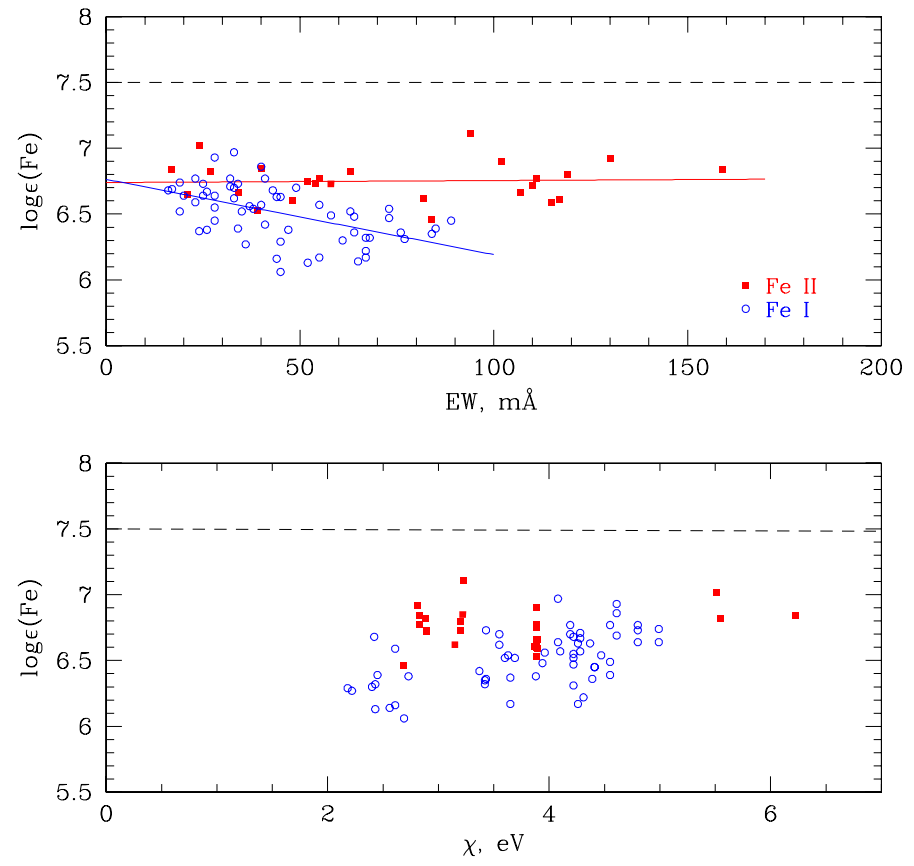

Fig. 3. Dependence of iron abundance in $\mathrm{BD}+46^{\circ} 442$ on EWs and the lower excitation potentials of individual lines for our preferred model atmosphere $T_{\text {eff }} / \log g / V_{\text {tur }}=6250 / 1.5 / 4.0 . T_{\text {eff }}$ was constrained from fits to hydrogen lines, $V_{\text {tur }}$ from the requirement of no trend of individual Fe II abundances with EWs, and $\log g$ from the agreement between the iron abundance inferred from Fe II and weak Fe I lines (strong Fe I lines being more affected by non-LTE). The lower panel is less conclusive owing to the non-uniform coverage of the excitation potentials by the Fe II lines. The dashed line indicates the iron abundance of the Sun.

flag to each element (each ionization state), that reflects the reliability of the final abundance estimate: flag 1 (best) - ions that do not display a trend with EW; flag 2- neutrals for which at least three lines with $\mathrm{EW} \leq 50 \mathrm{~m} \AA$ were available for averaging; flag 3 (least reliable) - all other cases. These flags are reflected in the circle sizes in Fig. 5. The errorbars are the rms values, they result from the uncertainties in the EW measurement and the values of $\log g f$-s. Table 1 also gives the systematic errors caused by the uncertainties in the model parameters. Singly ionized species are far more weakly sensitive to variations in $T_{\text {eff }}$ and $V_{\text {tur }}$ than the neutral ones, but more sensitive to $\log g$.

It is easy to see that there is a moderate metal deficiency in $\mathrm{BD}+46^{\circ} 442$, at the level of $-0.5 \ldots-0.7$ dex, relative to the Sun. Apart from the slightly more abundant $\mathrm{C}$ and $\mathrm{S}$, however, there is no obvious trend with $T_{\text {cond. }}$. The $\alpha$-process elements ( $\mathrm{Si}, \mathrm{S}, \mathrm{Mg}$, Ti) have a $0.1-0.3$ dex enhancement over the iron group, while s-process elements (Y, Zr, Ba, Nd) are not particularly enriched. The high abundance of $\mathrm{N}$ is likely a result of an evolutionary enrichment during the first and the second dredge-ups, but can also be affected by the non-LTE effects (Takeda 1992; Przybilla \& Butler 2001). Overall this is a typical composition of a thick disk star without chemical depletion (Reddy et al. 2006).

\section{Spectral energy distribution}

Figure 6 compares photometric observations of $\mathrm{BD}+46^{\circ} 442$ to the reddened photospheric Kurucz model with stellar parameters derived in Sect. 3.2. The total reddening of $E(B-V)=0.18$ was obtained by minimizing the difference between the adopted reddened model and the observed optical and near-infrared fluxes.

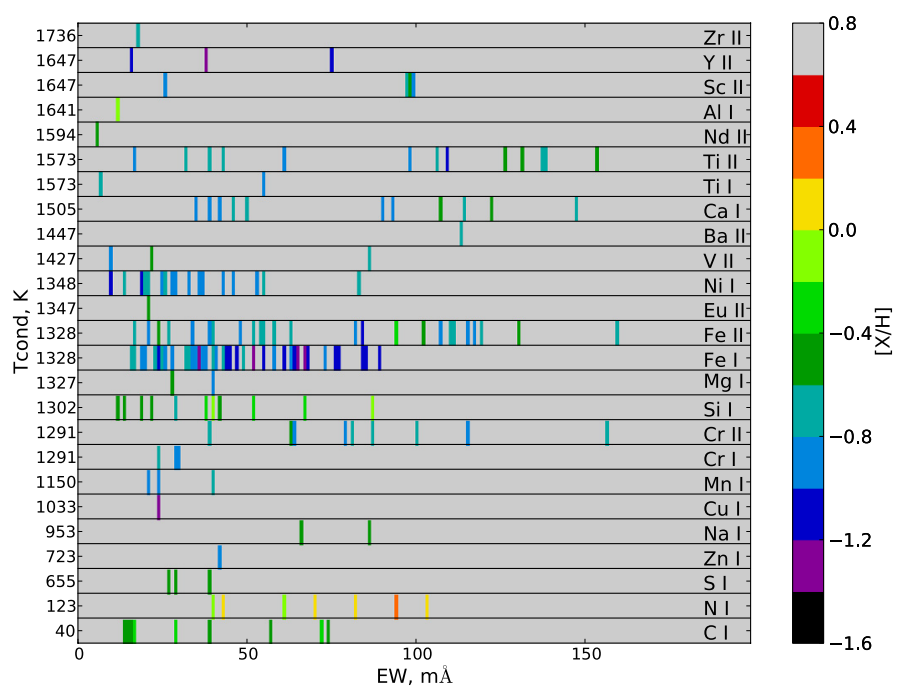

Fig. 4. Chemical composition of $\mathrm{BD}+46^{\circ} 442$ as inferred from the EW measurements of individual lines in the 17 August 2009 spectrum with the preferred model atmosphere $T_{\text {eff }} / \log g / V_{\text {tur }}=6250 / 1.5 / 4.0$. Colour-coded abundances are given relative to the Sun. Elements are arranged in the order of increasing condensation temperature.

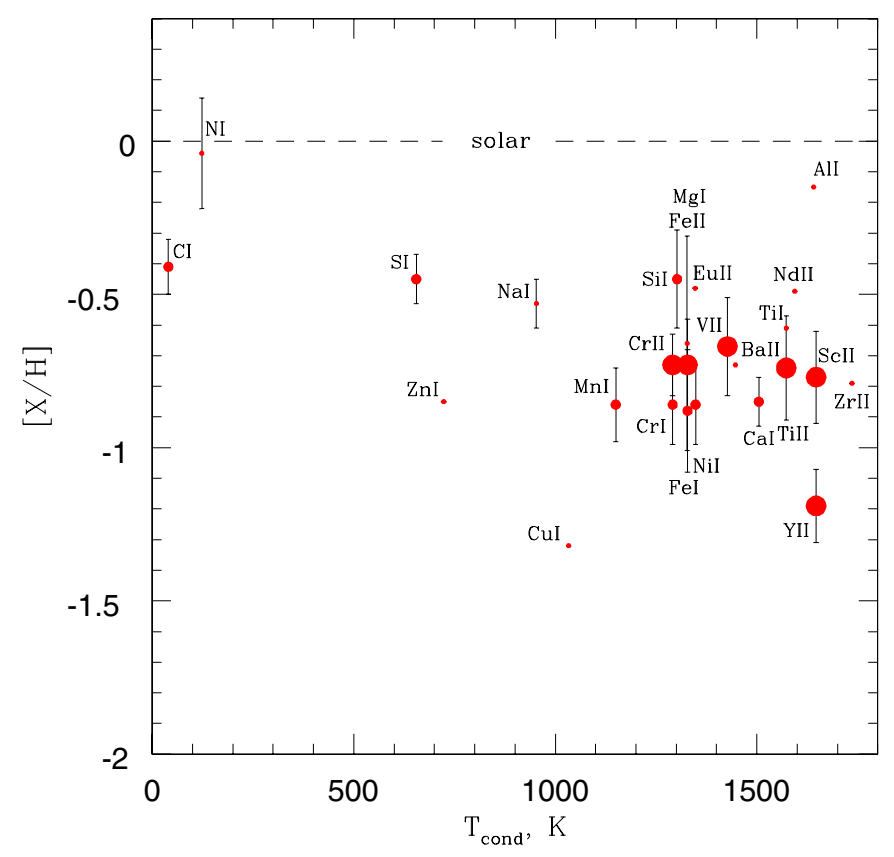

Fig. 5. Abundances of $\mathrm{BD}+46^{\circ} 442$ averaged from several lines of a given element, with errorbars representing the line-to-line rms scatter. The circle size designates the insensitivity of the lines to the non-LTE effects (the bigger the more reliable, see text). No significant depletion pattern is observed for this star; weak depletion is tentatively present, but better estimates are needed for the light elements to confirm this.

The presence of the excess emission beyond $2 \mu \mathrm{m}$ is obvious. When we selected $\mathrm{BD}+46^{\circ} 442$ for our sample, we could only rely on the IRAS detection of the excess (of which the $100 \mu \mathrm{m}$ flux is still an upper limit), and now we confirm this with the two other space missions, AKARI (Ishihara et al. 2010) and WISE (Wright et al. 2010). The SED, which is clearly not doublepeaked and only slightly reddened, is inconsistent with a simple detached shell distribution for the circumstellar material. The 
Table 1. Chemical composition of $\mathrm{BD}+46^{\circ} 442$.

\begin{tabular}{|c|c|c|c|c|c|c|c|c|}
\hline \multirow[t]{2}{*}{$\bar{Z}$} & \multirow[t]{2}{*}{ Ion } & \multirow[t]{2}{*}{$\log \epsilon$} & \multirow[t]{2}{*}{$\overline{[\mathrm{X} / \mathrm{H}]}$} & \multirow[t]{2}{*}{ rms } & \multicolumn{2}{|c|}{$\Delta[\mathrm{X} / \mathrm{H}]$} & \multirow[t]{2}{*}{$\bar{N}$} & \multirow[t]{2}{*}{ Flag } \\
\hline & & & & & $A$ & $\bar{B}$ & & \\
\hline 6 & $\mathrm{CI}$ & 8.11 & -0.41 & 0.09 & -0.05 & +0.05 & 6 & 2 \\
\hline 7 & N I & 7.89 & -0.04 & 0.18 & +0.02 & -0.01 & 2 & 3 \\
\hline 11 & $\mathrm{NaI}$ & 5.79 & -0.53 & 0.08 & -0.09 & +0.09 & 2 & 3 \\
\hline 12 & Mg I & 6.92 & -0.66 & 0.35 & -0.07 & +0.07 & 2 & 3 \\
\hline 13 & Al I & 6.32 & -0.15 & - & -0.09 & +0.09 & 1 & 3 \\
\hline 14 & $\mathrm{Si} \mathrm{I}$ & 7.09 & -0.45 & 0.16 & -0.08 & +0.07 & 8 & 2 \\
\hline 16 & S I & 6.67 & -0.45 & 0.08 & -0.06 & +0.06 & 3 & 2 \\
\hline 20 & $\mathrm{CaI}$ & 5.51 & -0.85 & 0.08 & -0.11 & +0.12 & 5 & 2 \\
\hline 21 & Sc II & 2.38 & -0.77 & 0.15 & -0.25 & +0.26 & 4 & 1 \\
\hline 22 & Ti I & 4.40 & -0.61 & - & -0.17 & +0.16 & 1 & 3 \\
\hline 22 & Ti II & 4.27 & -0.74 & 0.17 & -0.25 & +0.24 & 13 & 1 \\
\hline 23 & V II & 3.30 & -0.67 & 0.16 & -0.23 & +0.23 & 3 & 1 \\
\hline 24 & $\mathrm{Cr}$ I & 4.75 & -0.86 & 0.13 & -0.17 & +0.16 & 3 & 2 \\
\hline 24 & Cr II & 4.86 & -0.73 & 0.10 & -0.17 & +0.18 & 9 & 1 \\
\hline 25 & Mn I & 4.52 & -0.86 & 0.12 & -0.14 & +0.14 & 3 & 2 \\
\hline 26 & $\mathrm{Fe} \mathrm{I}$ & 6.59 & -0.88 & 0.20 & -0.13 & +0.13 & 39 & 2 \\
\hline 26 & Fe II & 6.75 & -0.73 & 0.15 & -0.18 & +0.19 & 25 & 1 \\
\hline 28 & $\mathrm{Ni} I$ & 5.37 & -0.86 & 0.13 & -0.14 & +0.13 & 15 & 2 \\
\hline 29 & $\mathrm{Cu} \mathrm{I}$ & 2.89 & -1.32 & - & -0.20 & +0.21 & 1 & 3 \\
\hline 30 & $\mathrm{ZnI}$ & 3.72 & -0.85 & - & -0.16 & +0.18 & 1 & 3 \\
\hline 39 & Y II & 1.03 & -1.19 & 0.12 & -0.27 & +0.27 & 3 & 1 \\
\hline 40 & Zr II & 1.79 & -0.79 & - & -0.25 & +0.25 & 1 & 3 \\
\hline 56 & Ba II & 1.40 & -0.73 & - & -0.31 & +0.30 & 1 & 3 \\
\hline 60 & Nd II & 1.07 & -0.49 & - & -0.28 & +0.29 & 1 & 3 \\
\hline 63 & Eu II & 0.02 & -0.48 & - & -0.26 & +0.27 & 1 & 3 \\
\hline
\end{tabular}

Notes. Abundances are given for our best-fit photospheric model $T_{\text {eff }} / \log g / V_{\text {tur }}=6250 / 1.5 / 4.0 . \Delta[\mathrm{X} / \mathrm{H}]$ designates abundance changes corresponding to the changes in $T_{\text {eff }} / \log g$ of $-250 \mathrm{~K} /-0.5$ dex (case A) and $+250 \mathrm{~K} /+0.5$ dex (case B), which are our second best atmospheric model estimates. The flag designates the quality of the $\mathrm{N}$ lines used in the averaging, from one for the best cases to three for the worst. Elements with flag 3 had fewer than three lines with $E W \leq 50 \mathrm{~m} \AA$.

SED is instead characteristic of a star surrounded by a passive disk (e.g, in De Ruyter et al. 2006; Gielen et al. 2007).

We modelled the SED of BD $+46^{\circ} 442$ with MCMax (Min et al. 2009), a two-dimensional Monte Carlo radiative transfer disk code. This code computes the temperature structure and density of the disk. The vertical scale height of the disk is computed by an iteration process, assuming vertical hydrostatic equilibrium. For the input stellar model, we use the parameters discussed in Sect. 3 and adopt a total system mass $M_{\star}=2 M_{\odot}$ and a typical post-AGB luminosity of $L_{\star}=2000 L_{\odot}$ (corresponding to a distance for the system of $3.3 \mathrm{kpc}$ ). Since we lack information on the exact mineralogy of the dust in the disk, we use a mixture of astronomical silicates and metallic iron, with grain sizes ranging from 0.1 to $100 \mu \mathrm{m}$. The adopted gas-to-dust ratio is kept fixed at 100 . The geometry of the disk is determined by the inner and outer radii $R_{\text {in }}$ and $R_{\text {out }}$, the total dust mass $M_{\text {dust }}$, and the power law of the surface-density distribution of the dust $\Sigma \sim R^{-p}$.

To ensure that the number of free parameters in the model are small, we keep the outer radius fixed at $R_{\text {out }}=500 \mathrm{AU}$, and use a value $0.5<p<2.0$ for the surface-density distribution, as expected in a disk environment. To account for the low observed total reddening, the inclination is kept fixed at $15^{\circ}$. This modelling is quite degenerate and equally well-fitting models with slightly different sizes, total masses, and surface-density distributions can be found. Complementary measurements, such as interferometric observations and infrared

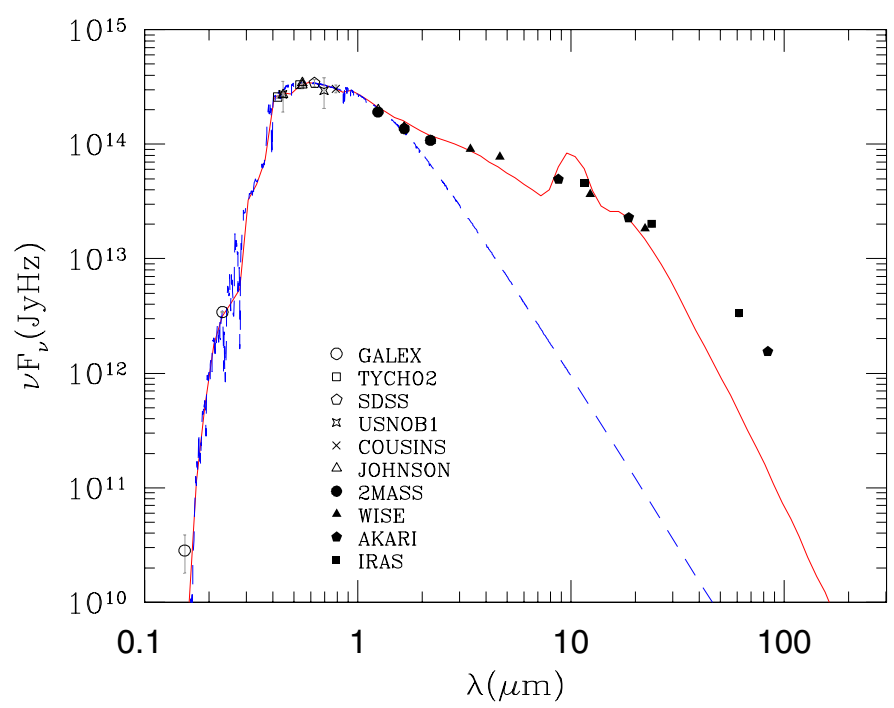

Fig. 6. The observed spectral energy distribution of $\mathrm{BD}+46^{\circ} 442$ plotted against the reddened Kurucz's model (dashed line) and our star+disk model (solid line). The flux up to $30 \mu \mathrm{m}$ can be reproduced with $10^{-6} M_{\odot}$ of dust in grains smaller than $100 \mu \mathrm{m}$ in size that reside in a ring that stretches from $7 \mathrm{AU}$ to $\sim 500 \mathrm{AU}$.

spectroscopy, would be invaluable to constrain further the disk geometry and mineralogy.

In Fig. 6, we show a disk model that closely describes the photometric data. The disk parameters for this model are $R_{\text {in }}=7 \mathrm{AU}, R_{\text {out }}=500 \mathrm{AU}, p=1.6$, and $M_{\text {dust }}=2 \times 10^{-6} M_{\odot}$. For the dust composition, we use a combination of amorphous olivine and metallic iron, in a ratio of $\sim 96 \% / 4 \%$. The AKARI and IRAS fluxes redwards of $30 \mu \mathrm{m}$ cannot be fitted by this model because that part of the SED requires even larger dust grains than adopted in the model. These large grains are likely not uniformly mixed in the disk, but must have settled towards the mid-plane. Modelling this process is beyond the scope of our investigation.

$\mathrm{BD}+46^{\circ} 442$ thus belongs to a distinct group of evolved stars surrounded by dusty disks (Gielen et al. 2011). To verify whether it is a binary system and determine whether the disk is either circumstellar or circumbinary, we analyse the radial velocities.

\section{Radial velocity curve}

We measured RVs using a cross-correlation method with a mask representing a G2 star. The mask consists of $\sim 1100$ lines. Crosscorrelation functions (CCFs) extracted from each order were averaged, and the resultant profile was fitted with a Gaussian to determine the RV. Some asymmetries in the CCF were noted that appear to correlate with the orbital phase (see discussion in the following section). They, however, were never large enough to affect the location of the main peak. The measured velocities are given in Table A.1 and plotted in Fig. 7. The errors in the RV consist of two quadratically added terms: the error in the Gaussian fit and the $0.2 \mathrm{~km} \mathrm{~s}^{-1}$ uncertainty due to the drift of the wavelength calibration zero-point.

As one can see from Fig. 7, the three seasons of observations reveal a regular variation in the $\mathrm{RV}$ with a period $\sim 100$ days and peak-to-peak amplitude of $\sim 40 \mathrm{~km} \mathrm{~s}^{-1}$. Integrating over half a period under the RV curve, we obtain a displacement of $130 R_{\odot}$, which is more than the radius of a post-AGB star. The radial velocity variations cannot be due to pulsations. 


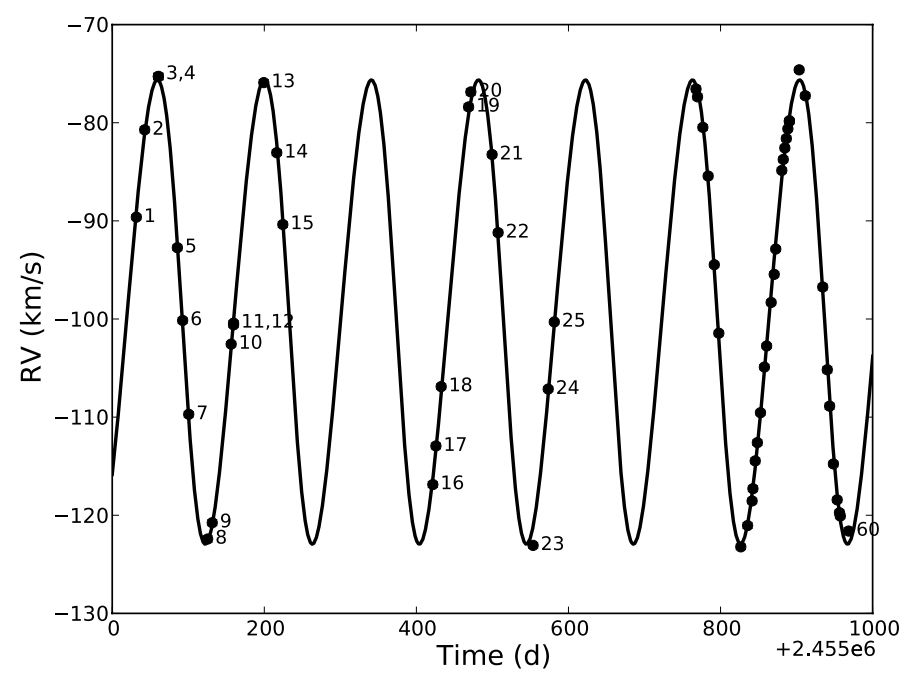

Fig. 7. HERMES radial velocities of $\mathrm{BD}+46^{\circ} 442$ plotted against Keplerian orbit with parameters listed in Table 2.

A long period with a nearly sinusoidal shape of the RV curve, near-constant temperature, and the large displacement of the photosphere firmly exclude pulsations. We must be instead observing an orbital motion around an invisible companion. $\mathrm{BD}+46^{\circ} 442$ is thus an SB1 system. We obtained a very good Keplerian solution with only one point (spectrum 23) out of 60 deviating by more than $3 \sigma$ (Fig. 8). The derived orbital parameters are given in Table 2. The errors in the parameters were estimated via the Monte Carlo method, using 1000 simulated $\mathrm{RV}$ sets. For a given date a value for the RV was drawn from a Gaussian distribution with a mean and $\sigma$ equal to the observed RV value and its error, respectively. For every simulated dataset the orbital parameters were obtained, and their scatter was used as an estimate of the error in the parameters obtained from the real data. An example application of this procedure for the orbital period and eccentricity is given in Fig. 9. As one can see, the eccentricity albeit small (0.08), significantly differs from zero.

Our observations provide the first account as far as we know of the RV variations in $\mathrm{BD}+46^{\circ} 442$, that we successfully reproduce with a Keplerian orbit. BD $+46^{\circ} 442$ proves to be yet another evolved system with a disk that is a binary. The semi-major axis of the primary is at most $1.2 \mathrm{AU}$ (for $i=15^{\circ}$ ). This is smaller than the silicate dust sublimation radius that would be at $2 \mathrm{AU}$ (given $L_{\star}=2000 L_{\odot}$ ), and much smaller than the actual inner disk radius at $7 \mathrm{AU}$ estimated from the fit to the NIR excess with metallic iron particles. This barely leaves space for a cicumstellar dusty disk around any of the binary components, unless the gas is very optically thick to shield some radiation. We conclude that the dusty disk producing the IR excess is circumbinary rather than circumstellar.

\section{Line profiles}

\section{1. $H \alpha$}

In Fig. A.1, we show that $\mathrm{H} \alpha$ alternates between two major profiles: a double-peak emission line with peaks separated by $\sim 100 \mathrm{~km} \mathrm{~s}^{-1}$ and wings stretching to $\pm 200 \mathrm{~km} \mathrm{~s}^{-1}$, and a P Cyg-like profile. This is better illustrated in Fig. 10 (medium left panel) where we depict $\mathrm{H} \alpha$ after subtraction of the model photospheric profile. The resulting profile contains both emission and absorption components that vary in strength
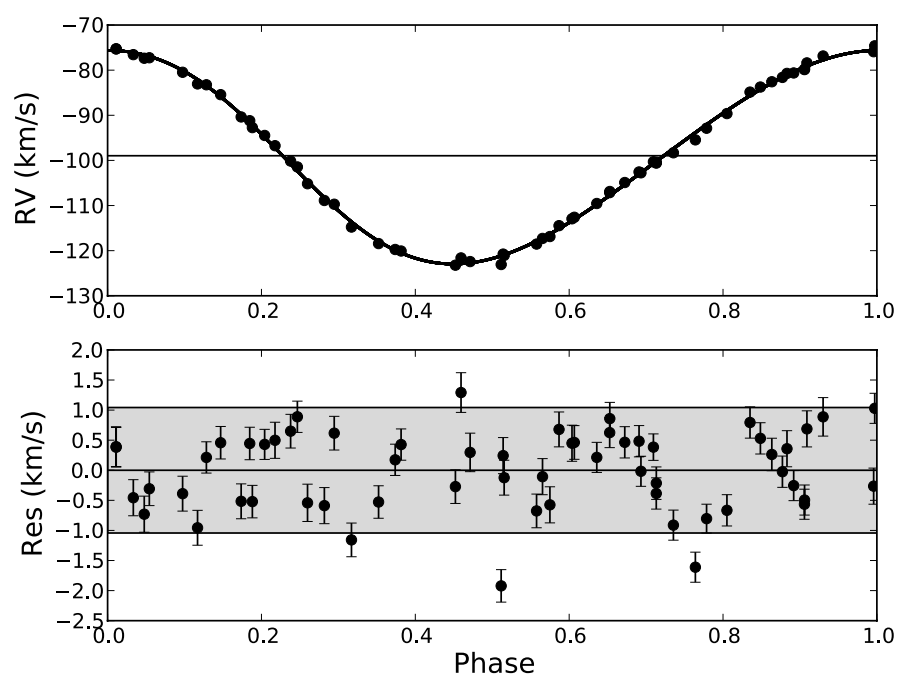

Fig. 8. Upper panel: radial velocities of $\mathrm{BD}+46^{\circ} 442$ phased with a period of 140.77 days and overlaid on our Keplerian fit. The horizontal line marks the corresponding systemic velocity. The $\phi=0$ was defined to correspond to the maximum RV (i.e. near one of the elongations). Bottom panel: residuals from the fit with $\pm 3 \sigma$ margins.
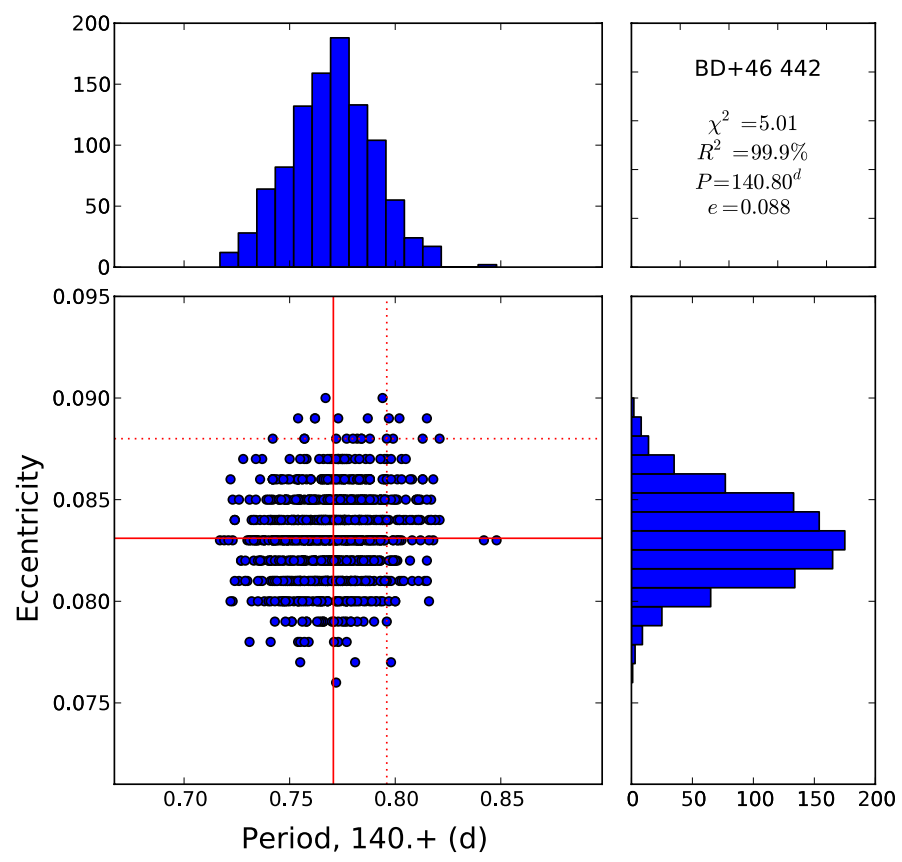

Fig. 9. Distribution of the orbital period and eccentricity values obtained from 1000 simulated RV data sets. The solid cross marks the solution based on the observed RVs (see Table 2), while the dotted cross and the upper right panel show an alternative solution with a slightly better $\chi^{2}$ derived from the simulated RV data sets.

with the orbital phase. The double-peak emission is observed most of the time, but is strongest and most symmetric between $\phi=0.7-0.8$, corresponding to the giant's inferior conjunction (when it is between us and the companion, Fig. 11). When the giant starts to retreat, the emission diminishes, while the central absorption blue-shifts and broadens, quickly turning into a $\mathrm{P}$ Cyg profile. The expansion velocities reach $350 \mathrm{~km} \mathrm{~s}^{-1}$ at $\phi=0.3-0.4$ when the companion is in front. Higher members of the Balmer series develop broadened cores at this phase, but the effect is much less pronounced than in $\mathrm{H} \alpha$, particularly in the wings (Fig. A.1), which justifies their use for estimating 

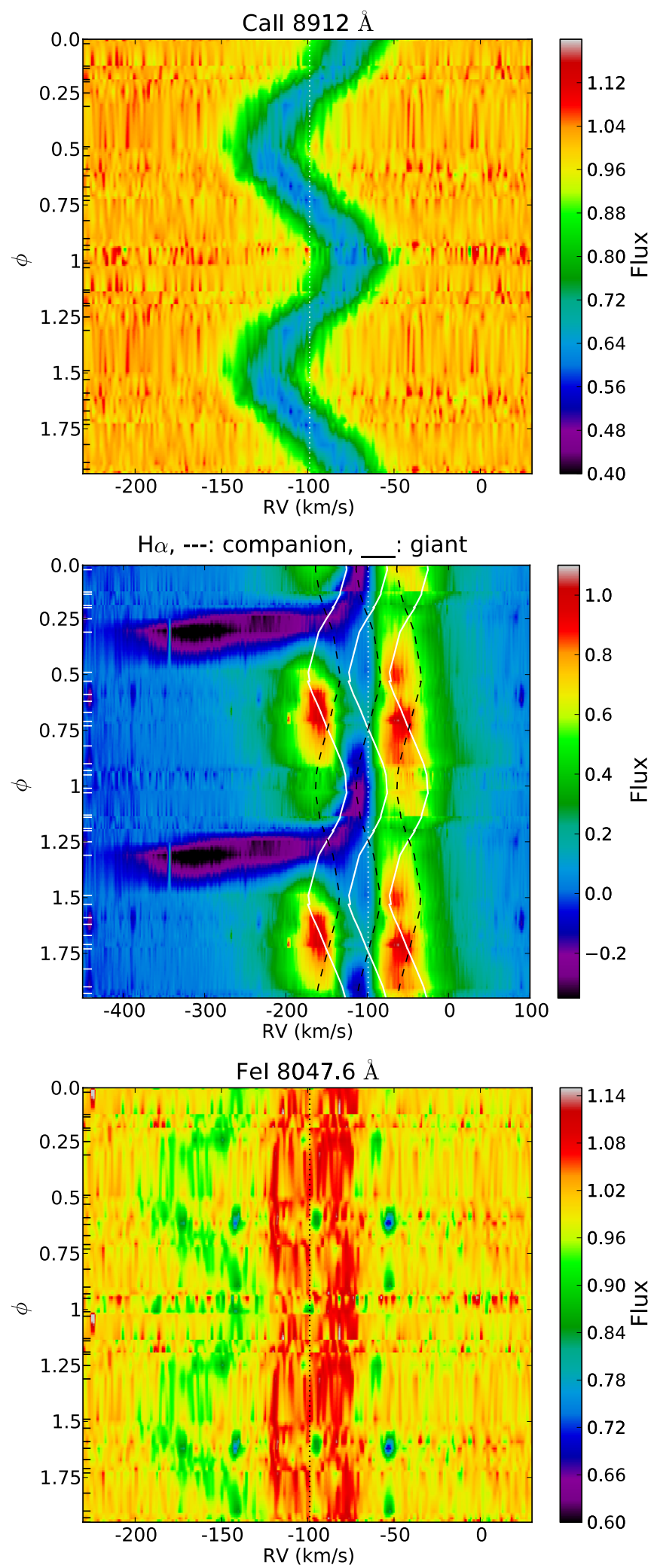

Fell $5169 \AA$
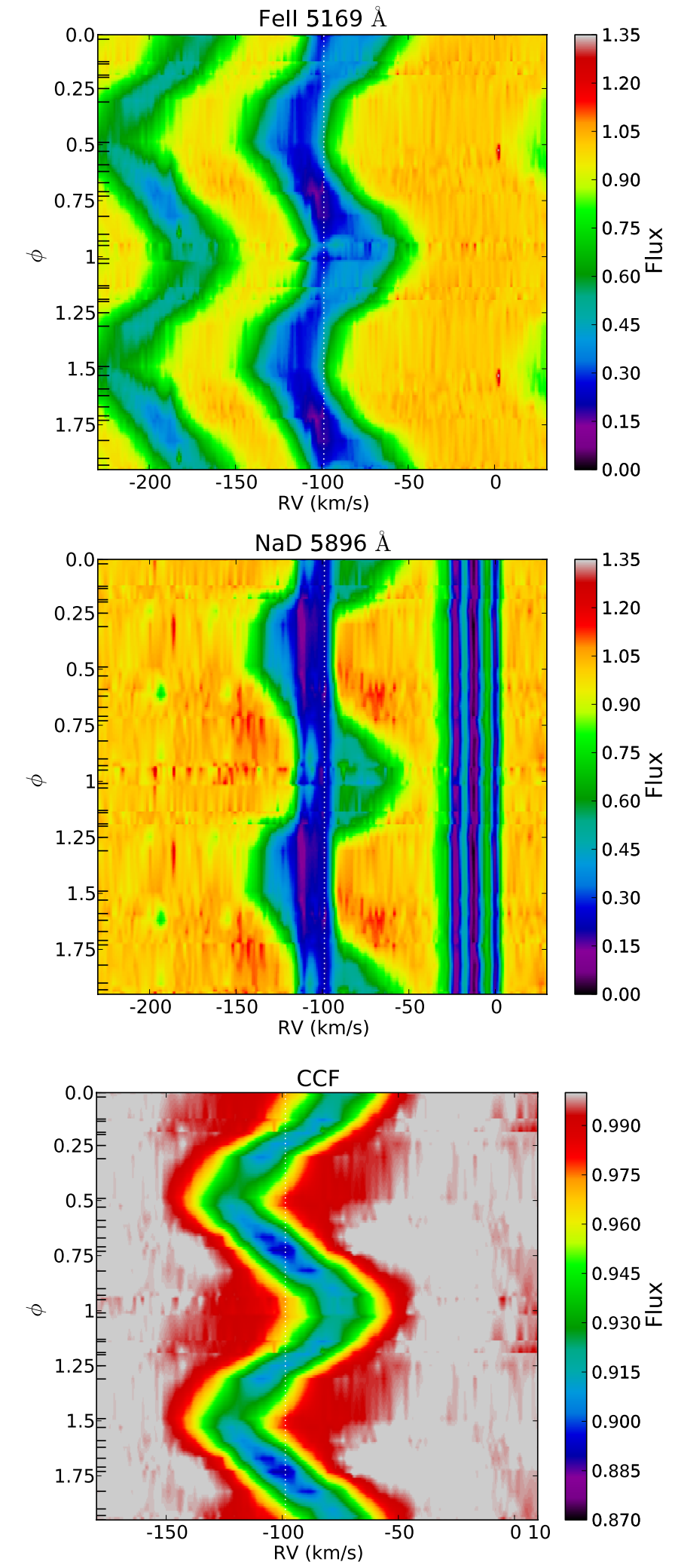

Fig. 10. Dynamic spectra of selected lines and the CCF as a function of the orbital phase (time runs from top to bottom). Colours designate continuum-normalized fluxes (except for $\mathrm{H} \alpha$ ), with black corresponding to the strongest absorption. Fluxes in the missing phases were obtained by a linear interpolation between the nearest observed phases. Dotted vertical line marks the systemic velocity, while the horizontal dashes on the left indicate the observed phases. One orbital period is shown twice to guide the eye. $\mathrm{H} \alpha$ is plotted after subtraction of the photospheric model spectrum; solid and dashed lines represent the RV curves (original and $\pm 50 \mathrm{~km} \mathrm{~s}^{-1}$ offset) of the giant and of the putative companion to illustrate the lack of significant motion in the emission component. Only data from the first two seasons of observations are shown (spectra 1-25, obtained between July 2009-January 2011); later observations confirm the general behaviour with phase, but the intensities of the circumstellar features appear to vary slightly from cycle to cycle. 
Table 2. Orbital elements of BD $+46^{\circ} 442$.

\begin{tabular}{lcc}
\hline \hline Parameter & Value & $\sigma$ \\
\hline$P(\mathrm{~d})$ & 140.77 & 0.02 \\
$a \sin i(\mathrm{AU})$ & 0.31 & 0.001 \\
$f(m)\left(M_{\odot}\right)$ & 0.19 & 0.001 \\
$K\left(\mathrm{~km} \mathrm{~s}^{-1}\right)$ & 23.66 & 0.06 \\
$e$ & 0.08 & 0.002 \\
$\omega\left({ }^{\circ}\right)$ & 100 & 2 \\
$T_{0}(\mathrm{JD})$ & 2455094.6 & 0.6 \\
$\gamma\left(\mathrm{km} \mathrm{s}^{-1}\right)$ & -98.96 & 0.04 \\
$\chi^{2}$ & 6.4 & \\
$R^{2}$ & $99.83 \%$ & \\
\hline
\end{tabular}

Notes. Orbital parameters with their uncertainties and two statistical parameters indicating the goodness of the RV fit (reduced chi-squared and the coefficient of determination).

$T_{\text {eff }} / \log g$. The $\mathrm{H} \alpha$ profile as a whole does not seem to shift in $\mathrm{RV}$, but is not centred on the systemic velocity $\left(V_{\text {syst }}\right)$ either, rather, it is permanently blue-shifted by $\sim 14 \mathrm{~km} \mathrm{~s}^{-1}$.

\subsection{Non-photospheric components of metal lines}

In the other panels of Fig. 10 (and in Fig. A.2), we show the $\mathrm{CCF}$ and a representative set of metal line profiles. As expected, the CCF follows the behaviour of weak to medium-strong lines, such as Ca II $8912 \AA$, that constitute the majority of the unblended lines in the CCF list. These lines are "well behaved" because they are symmetric and hardly variable, except for the RV that reflects the orbital motion of the giant. The Fe II(42) triplet, on the other hand, is an example of strong lines $(E W \gtrsim 300 \mathrm{~m} \AA)$ that have an additional narrow component near $V_{\text {syst }}$. This can be most clearly seen when the giant retreats from us $(\phi=0.9-1.1)$, while in the opposite phase it appears less prominent, being either weaker or blue-shifted (by $\sim 10 \mathrm{~km} \mathrm{~s}^{-1}$ from $V_{\text {syst }}$ ) and overlapping with the photospheric component. Other similar cases involve the strong lines of Ba II, Ca II, Fe I, Fe II, Mg I, and Ti II, and the resonance lines of $\mathrm{KI}$. Exceptions include the prominent O I triplets at $7775 \AA$ and $8446 \AA$, and the Ca II doublet at $8915 \AA$, which have normal, single-deep, profiles. These transitions, however, are produced by much higher excitation $\left(\chi_{\text {low }}=\right.$ $7-9 \mathrm{eV})$ and form deeper in the atmosphere. The strengthening of the central absorption between $\phi=0.8-1.25$ and the disappearance or blue-shift between $\phi=0.3-0.7$ resemble the behaviour of the central absorption in $\mathrm{H} \alpha . \mathrm{H} \alpha$ absorption, however, is more blue-shifted, particularly in the P Cyg phase.

The Na I D lines also show a central absorption component, but also an additional, narrower one $13 \mathrm{~km} \mathrm{~s}^{-1}$ to the blue from $V_{\text {syst }}$, which coincides with the centroid of $\mathrm{H} \alpha$. Both components are probably of circumstellar origin, but it is difficult to study them owing to blending with the photospheric lines. To the red, there are three supposedly ISM components, at RV $=-23$, -13 , and $-1 \mathrm{~km} \mathrm{~s}^{-1}$, that are stable in RV and depth. They are also observed in the resonance K I lines. Using a code provided in Reid et al. (2009), we calculated kinematic distances to the foreground clouds responsible for these components. The largest distance is obtained for the most blue-shifted component $\left(V_{\text {helio }}=-23 \mathrm{~km} \mathrm{~s}^{-1}, V_{\mathrm{LSR}}^{\mathrm{rev}}=-18 \mathrm{~km} \mathrm{~s}^{-1}\right)$, which is $1.25 \pm$ $0.5 \mathrm{kpc}$. Taking this as a minimum distance to BD+46 442 itself, and correcting for the reddening $A_{\mathrm{V}}=0.56$, we obtain a lower limit to the stellar luminosity of $M_{\mathrm{V}}=-1.54_{-0.81}^{+1.19}$. The star is clearly brighter than normal FG giants of the luminosity class III $\left(M_{\mathrm{V}}=1 \ldots 2\right)$, but is consistent with being an $\mathrm{F}$ type
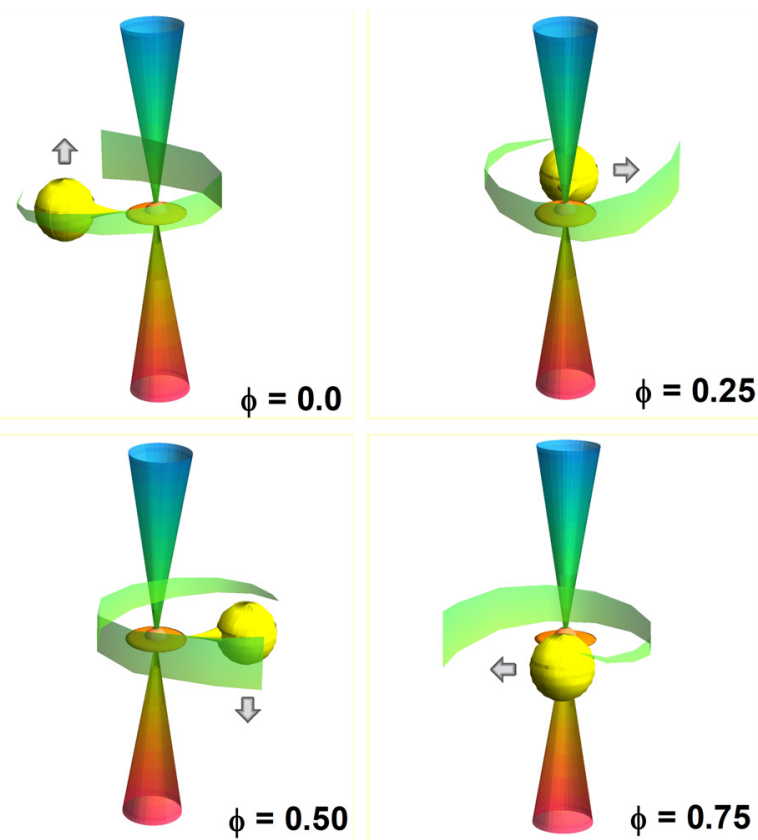

Fig. 11. Schematic representation (not to scale) of the views of $\mathrm{BD}+46^{\circ} 442$ at different orbital phases. The dusty circumbinary disk has been omitted to enable us to more clearly illustrate gas flows in the vicinity of the components (the inner disk wall would be in all cases outside the boundaries of the figure). The inclination, both of the orbit and of the likely precessing jet, remains to be constrained. The arrow shows the direction of the giant's motion. The figure illustrates how an accretion jet around the companion can explain the transient P Cyg-like profile of $\mathrm{H} \alpha$, while a gas stream trailing behind the giant can explain the narrow absorption component of metal lines.

post-AGB star, which have $M_{\mathrm{V}}=-2 \ldots-3$ (Bond 1997; Ginestet \& Carquillat 2002). On the other hand, the two components at $V_{\text {helio }} \sim-100 \mathrm{~km} \mathrm{~s}^{-1}$ would indicate an Iab supergiant $\left(M_{\mathrm{V}}=-6 \ldots-7\right)$ at more than $2.5 \mathrm{kpc}$ above the Galactic plane, which is very unlikely. Thus, the two components near $V_{\text {syst }}$ are most likely circumstellar.

In addition, as can be seen from Fig. 10 , near $\phi=0.75$ corresponding to the giant's inferior conjunction, weak emission appears in the wings of the sodium lines that resembles that of $\mathrm{H} \alpha$. We observe only one other system of emission lines besides $\mathrm{H} \alpha$ and Na I D: the weak Fe I(12) multiplet at 8047.6 $\AA$ and 8075.1 $\AA$. As for to the central absorption of metal lines, this emission is centred on the systemic velocity and does not contribute to the orbital motion (Fig. 10).

\subsection{Secondary spectrum?}

The metal lines noticeably strengthen near the giant's inferior conjunction $(\phi=0.75)$. Zooming in on the wings of the CCF (see the right-bottom panel of Fig. 10), however, also reveals faint absorption spectrum moving in anti-phase with the giant's spectrum. Therefore, we may instead be observing a companion spectrum. We remodelled the CCF profiles with two Gaussians, where possible, and obtained a semi-amplitude of $15 \pm 5 \mathrm{~km} \mathrm{~s}^{-1}$ for the secondary spectrum. Dividing by the $23.7 \mathrm{~km} \mathrm{~s}^{-1}$ of the primary, we obtain a mass ratio of 0.6. Given this mass ratio and the mass function of 0.2 , we obtain the individual masses of $0.9-0.3 M_{\odot}$ for the giant and 1.4-0.5 $M_{\odot}$ for the companion for a range of inclinations of $45-90^{\circ}$. These masses are consistent with a low-mass pair where the originally more 
massive component has evolved into a post-AGB, while the companion is either still on the MS or in the sub-giant stage. Using expressions for the Roche-lobe radius from Paczyński (1971), that depend only on the mass ratio, we obtain for the giant $R_{1} \approx 0.37 \times\left(a_{1}+a_{2}\right)=57-39 R_{\odot}$. The radius of $\mathrm{BD}+46^{\circ} 442$ matches this range well if it has a typical post-AGB luminosity of 2000-3000 $L_{\odot}$. Therefore, we are observing an interacting system where the post-AGB star is (close to) filling its Roche-lobe, hence feeding a more compact companion.

We stress, however, that these are very preliminary estimates. The depth of the secondary component in the CCF is about $1 \%$ (compared to $10 \%$ of the giant's spectrum), which is far lower than the typical $\mathrm{S} / \mathrm{N}$ of our spectra. The presence of this component thus cannot be verified using single lines. Furthermore, the spectral type of the companion cannot differ significantly from that of the primary, otherwise one would not detect it with the G2 mask. The companion should then be only ten times fainter than the giant primary, which is too bright for it to be a MS star. And if both components were giants, they would have to have nearly identical initial masses owing to the shortness of the giant stage. It is possible instead that we observe a pseudophotosphere of an accretion disk around the companion rather than the companion's spectrum itself. Higher $\mathrm{S} / \mathrm{N}$ spectra and a multi-band photometry are needed to confirm the amount of the contribution of the companion to the system's flux.

\section{Discussion}

The discovery of $\mathrm{RV}$ variations in $\mathrm{BD}+46^{\circ} 442$ provides strong support for the disk-binary connection in post-AGB stars. Its period of $140.77 \pm 0.02^{\mathrm{d}}$ is one of the shortest known for postAGB binaries. Its small but significant eccentricity indicates that the system is incompletely circularized. In the following discussion, we demonstrate how the observed spectral features in $\mathrm{BD}+46^{\circ} 442$ are indicative of ongoing mass transfer from the giant to the companion via the Roche-lobe overflow.

\section{1. $H \alpha$ variability in interacting binaries}

The $\mathrm{H} \alpha$ profiles discussed here are not uncommon among postAGB stars (Waters et al. 1993b; Pollard et al. 1997; Maas et al. 2005; Sánchez Contreras et al. 2008), but their variabilities have not been systematically studied as a function of orbital phase. We are aware of four other disk systems in which strong blueshifted absorption was noted to develop during the giant's superior conjunction: HR 4049 with $P_{\text {orb }}=430^{\mathrm{d}}, a \sin i=0.6 \mathrm{AU}$, $i \geq 60^{\circ}$, and $e=0.3$ (Waelkens et al. 1991; Van Winckel et al. 1995; Bakker et al. 1998); HD 44179 (the central star of the Red Rectangle nebula) with $P_{\text {orb }}=318^{\mathrm{d}}, a \sin i=0.5 \mathrm{AU}$, $i$ "effective" $=35^{\circ}$, and $e=0.4$ (Waelkens et al. 1996; Witt et al. 2009); IRAS 08544-4431 with $P_{\text {orb }}=508^{\mathrm{d}}, a \sin i=0.4$ AU, $i \sim 60^{\circ}$, and $e=0.2$ (Maas et al. 2003; Deroo et al. 2007; Van Winckel et al. 2009); and IRAS 19135+3937 with $P_{\text {orb }}=$ $127^{\mathrm{d}}, a \sin i=0.2 \mathrm{AU}$, and $e \sim 0.3$ (Gorlova et al. 2012). That $\mathrm{BD}+46^{\circ} 442$ has a very small eccentricity $(e=0.083 \pm 0.002)$ indicates that the cause of the spectral variations most likely lies in the varying line of sight towards the components, rather than in physical changes that occur during the orbital motion, such as greater mass loss at periastron.

The most common interpretations of the double-peak $\mathrm{H} \alpha$ emission in stellar spectra are: an inclined Keplerian gaseous disk (e.g. in Be and T Tau stars), the giant's atmosphere being irradiated by a hot companion (cataclysmic variables, symbiotics), a propagating shock in pulsating stars (Miras, RV Tau stars), and the chromosphere. We note that there is no indication in our spectra of a hot companion (neither helium lines nor nebular emission), that pulsations are excluded based on the smooth orbital RV curve, and that the existence of chromospheric activity has yet to be established in post-AGB stars. We therefore concentrate on the disk hypothesis. The emission may originate in the gaseous extension of the circumbinary dusty disk towards the centre of the system or in a circumstellar disk around one of the components. To distinguish the most likely of these possibilities, we searched for similarities with other types of interacting binaries. There, a disk sometimes forms around a more compact companion as the result of the Roche-lobe overflow or wind accretion from a larger companion.

In Algols, when the binary separation is large, the accretion stream from a cool giant curves and settles into a disk around a more massive hot MS primary (Richards \& Albright 1999). The simulations of Miller et al. (2007) show that the dominant source of $\mathrm{H} \alpha$ emission in Algols is the disk, while the stream provides a much smaller contribution. A similar situation may be occurring in $\mathrm{BD}+46^{\circ} 442$, with the exception that the accreting companion is much fainter than the giant (Fig. 11). The problem with this interpretation of the $\mathrm{H} \alpha$ emission is that there is a lack of significant motion in anti-phase with the absorption spectrum of the primary (Fig. 10). The P Cyg-like profile, on the other hand, is a signature of an outflow. It is very rare to find such a broad absorption feature (with velocities up to $-300 \mathrm{~km} \mathrm{~s}^{-1}$ ) in Algols, and it may not be observed in every cycle (Peters 1989; Miller et al. 2007). Algols usually display classical and inverse P Cyg-like profiles at a smaller range of velocities just before and after the giant's inferior conjunction, which is explained by the disk eclipse. This is clearly not the case in BD $+46^{\circ} 442$ where the wind profile reaches its strongest velocity near the giant's superior conjunction. An explanation of the P Cyg profile, therefore, should be found elsewhere.

W Ser systems are thought to correspond to the stage preceding Algols, when the transfer rate is very high and the accretion disk completely obscures the accretor. In at least one such system, $v$ Sgr with $P_{\text {orb }}=137^{\mathrm{d}}$ (Netolický et al. 2009), a P Cyg-like profile is observed near the giant's superior conjunction, which Nariai (1967) explained as a "coronal stream" from the giant to the companion. At this orbital phase, the stream is directed towards us and at the same time is projected against the giant (Fig. 11), creating blue-shifted absorption. Furthermore, in the simulations of interacting binaries the accretion stream is often found to be curved in the direction of the giant's motion, which could explain why the highest speeds in $\mathrm{BD}+46^{\circ} 442$ are reached at a slightly later phase after the conjunction (compare spectra 6 and 7 in Fig. A.1). Interestingly, $v$ Sgr is considered to be a noneclipsing type of W Ser stars. In classical, edge-on, systems the blue-shifted absorption is not observed, perhaps because the accretion stream, when projected against the donor, is hidden from our view by the accretion disk.

Symbiotic stars are another relevant class of objects to consider. Their double-peaked emission profiles are quite common (Van Winckel et al. 1993), and are interpreted as either due to an accretion disk around a WD (Robinson et al. 1994; Van Eck $\&$ Jorissen 2002) or a disk wind (Skopal 2006). There are even reports that the blue peak gets suppressed at the orbital phase corresponding to the occurrence of the $\mathrm{P}$ Cyg-like profile in BD+46²42 (Robinson et al. 1994; Mürset et al. 2000), although the true broad absorption in symbiotics is only observed in outbursts. 


\subsection{Jet launching in the accretion disk of the companion}

To summarize, the variations in the $\mathrm{H} \alpha$ profile in $\mathrm{BD}+46^{\circ} 442$ are complex, but can be decomposed into several time-dependent properties that we need to explain: 1) the double-peak emission is rather stable in terms of velocity along the orbit, which means that it is connected to the system itself and not to one of the components. The emission, however, varies in terms of strength, being strongest during the giant's inferior conjunction; 2) the P Cyg-like absorption component is observed at very high velocity, but only during a very short orbital phase interval, when the giant is at the superior conjunction (see Fig. 10). As we have seen in the previous section, some of these aspects can be explained by the interaction with the companion.

It is useful to compare this behaviour to the line variability observed and discussed in HD 44179 by Witt et al. (2009), who attribute both emission and absorption in $\mathrm{H} \alpha$ to the same structure, namely a jet launched from the accretion disk of the companion. The P Cyg-like profile forms when the blue-shifted lobe is projected against the face of the giant, which is impossible to achieve for the red-shifted lobe, which is therefore always observed in emission. The jet launched in a precessing accretion disk around the companion of HD 44179 may have created the Red Rectangle nebula (Velázquez et al. 2011). This model can be adopted for $\mathrm{BD}+46^{\circ} 442$ (Fig. 11), with one caveat. The Red Rectangle is a peculiar system because its optical flux is dominated by scattering as our line of sight is in the orbital plane of the binary, allowing us to see both jets at a range of inclinations. For BD $+46^{\circ} 442$, in contrast, the smaller IR excess indicates that we observe the star directly, along the line-of-sight that is not in the orbital plane. The small amount of line-of-sight reddening shows that our aspect angle towards the system does not pass through the puffed-up circumbinary disk. The blue absorption seen at conjunction is created by the blue-shifted jet, which is projected onto the giant only in this specific orbital phase. Continuum photons coming from the giant are scattered outside the line-of-sight by $\mathrm{H} \alpha$ resonant scattering by high velocity hydrogen atoms in the jet. The Doppler shift of $300 \mathrm{~km} \mathrm{~s}^{-1}$ corresponds to the line-of-sight velocity component and needs to be deprojected to obtain the outflow velocity of the jet. As we constrain neither the jet opening angle, nor the angle between the jet axis of symmetry and the orbital plane, the deprojected velocity is difficult to quantify. The static double-peak emission must have an independent origin, as discussed in the previous section.

The existence of jets has been strongly advocated based on other independent arguments in e.g., a $\beta$ Lyr system (Harmanec 2002; Ak et al. 2007), a proto-type of the massive Rocheoverflow binaries (of which W Ser is a sub-class). Jets have been invoked to explain bipolar planetary and proto-planetary nebulae (PPNe), including the Red Rectangle (Cohen et al. 2004; Velázquez et al. 2011). Sahai et al. (2002) detected the proper motion of the jets in the PPNe Henize 2-90 at a velocity of a few hundred $\mathrm{km} \mathrm{s}^{-1}$, which is consistent with the Keplerian velocity at the surface of a low- to intermediate-mass MS star. Finally, jets have also been mentioned in the context of symbiotics and their symmetric bumps at $\pm 1000 \mathrm{~km} \mathrm{~s}^{-1}$ appearing in outbursts (Skopal et al. 2009), though they have never been invoked to explain the double-peaked profile of the quiescent state. It is difficult to include jets in either the hydrodynamic calculations because of the supersonic velocities, or the radiative transfer calculations. Nevertheless, the possibility of a jet contribution to either $\mathrm{H} \alpha$ and $\mathrm{H} \beta$ formation has been demonstrated by several studies (Budaj \& Richards 2004; Arrieta et al. 2005).

\subsection{Gas stream}

Further phenomenological similarity between $\mathrm{BD}+46^{\circ} 442$ and the mass-exchanging binaries can be seen in the nonphotospheric "shell" components of metal lines. The narrow features, mostly in absorption, discussed in BD $+46^{\circ} 442$ (such as the Fe II (42) and the near-IR Ca II triplets) were first noted in rapidly rotating hot stars (Slettebak 1986). They originate from the meta-stable levels at $2-4 \mathrm{eV}$ above the ground state, hence must form in a low-density, but hot (several kK) circumstellar medium. In cooler stars, shell components have mostly been studied in the resonance $\mathrm{Ca}$ II H\&K and $\mathrm{Na}$ I D lines, but these transitions are prone to interstellar contamination. Because of their corellation with the orbital phase of $\mathrm{BD}+46^{\circ} 442$, we again assume that they originate in the binary flows, rather than in some clumps in the circumbinary disk (as proposed for the protoplanetary disks, see e.g. Mora et al. 2004). The behaviour of these features appears to be very diverse from system to system, so it is unsurprising that they have been ascribed to a whole range of accretion-related structures: disk, disk-wind collision shocks, jets, streams, etc. (e.g. Andersen et al. 1988; Plavec 1988; Weiland et al. 1995; Miller et al. 2007; Sudar et al. 2011; Mennickent \& Smith 2010; Harmanec 2002; Quiroga et al. 2002).

We found that there is a particularly good resemblance between the behaviour of the metal absorption lines in BD $+46^{\circ} 442$ and those of the He I lines in a W Ser-type star RY Sct $\left(P_{\text {orb }}=\right.$ $11^{\mathrm{d}}$, orbital separation $\left.\sim 0.2 \mathrm{AU}\right)$, as described in Grundstrom et al. (2007). To explain these features, one needs to project gas onto the giant over at least half of the period, that moves with a slower velocity, for example a circumbinary Keplerian disk. Furthermore, this ring must have an asymmetric density distribution to explain the unequal strengths of the absorption at different elongations. Grundstrom et al. (2007) proposed that this structure could be a plume on the trailing side of the giant, namely an outflow from the L2 point that extends beyond the orbital plane and warps around the system (Fig. 11). This would explain why the central absorption is clearly visible when the giant recedes from us, but is much less pronounced in the opposite elongation. This outflowing arm is consistently reproduced in hydrodynamical simulations of interactive binaries (De Val-Borro et al. 2009; Mohamed \& Podsiadlowski 2011). Grundstrom et al. (2007) further speculate that the outflows through the L2 and L3 points could explain the formation of the large nebula $(R \sim 2000 \mathrm{AU})$ surrounding RY Sct. This scenario raises the intriguing possibility of the current formation of the circumbinary disk around $\mathrm{BD}+46^{\circ} 442$. Finally, in a recent study, Thomas et al. (2011) reports on the identical behaviour of some metal lines, including Fe II $4924 \AA$, in HD 44179. Unlike Grundstrom et al. (2007), they interpret it in terms of the photospheric line asymmetries produced by matter outflowing from the giant near periastron and falling back near apastron. This model, however, is questionable for BD $+46^{\circ} 442$ owing its low eccentricity. Observations of more systems with different eccentricities and orbit orientation are obviously needed to test these hypotheses.

Another manifestation of the circumstellar gas around $\mathrm{BD}+46^{\circ} 442$ are the two weak emission lines of the Fe I(12) multiplet in the far red region of the optical spectrum. These emission lines have been reported in at least two other disk postAGB sources, 89 Her (a binary with $P_{\text {orb }}=288^{\mathrm{d}}, a \sin i=$ $0.1 \mathrm{AU}, i=15^{\circ}, e=0.2$, and a resolved bipolar $\mathrm{CO}$ outflow, see Waters et al. 1993a; Bujarrabal et al. 2007) and a candidate RV Tau star QY Sge (Kameswara Rao et al. 2002). In $89 \mathrm{Her}$, these lines are narrow $\left(F W H M<10 \mathrm{~km} \mathrm{~s}^{-1}\right)$ and 
single-peaked, while in QY Sge and BD $+46^{\circ} 442$ they are much broader $\left(F W H M \sim 40 \mathrm{~km} \mathrm{~s}^{-1}\right)$ and perhaps even double-peaked. Unfortunately, the latter is difficult to measure accurately for $\mathrm{BD}+46^{\circ} 442$ owing to contamination by telluric absorption. Kameswara Rao et al. (2002) proposed that this emission originates in the bipolar wind from the system, in particular, from the gas within the inner cavity of the circumbinary disk that reemits starlight scattered by the disk walls. Assuming a total stellar mass for $\mathrm{BD}+46^{\circ} 442$ of $1.5 M_{\odot}$ and a circumbinary gas disk with Keplerian rotation, the HWHM $=20 \mathrm{~km} \mathrm{~s}^{-1}$ indicates a distance of 3.3 AU. Given that the inner radius of the dusty disk is at $7 \mathrm{AU}$, as deduced from the SED fitting, this gas may indeed be located within the inner disk hole. Kameswara Rao et al. (2002) also explained the broader emission in $\mathrm{H} \alpha$ and the $\mathrm{NaI} \mathrm{D}$ lines as originating in the same wind, only further out from the disk plane where the wind has been accelerated. This scenario would explain the lack of the RV variations in the emission lines of $\mathrm{BD}+46^{\circ} 442$, including $\mathrm{H} \alpha$. The remaining differences between both 89 Her and QY Sge and BD $+46^{\circ} 442$ could be due to their different inclination angles. To constrain the inclinantion angle of $\mathrm{BD}+46^{\circ} 442$, an accurate light-curve would be needed.

The next obvious step to confirm the association of all these circumstellar features with binarity, would be the systematic comparison of disk sources to the sources with spherical shells (as inferred from the SED, e.g. Van Aarle et al. 2011), as the latter presumably harbour single stars.

\section{Summary}

We have obtained 60 echelle spectra over a period of 3.5 years for $\mathrm{BD}+46^{\circ} 442$, which had been a poorly studied high latitude Galactic giant with a dusty disk. We derived the photospheric parameters $T_{\text {eff }}=6250 \pm 250 \mathrm{~K}, \log g=1.5 \pm 0.5$, and an average metallicity $[\mathrm{M} / \mathrm{H}]=-0.7 \pm 0.2$, without a strong depletion pattern. The enhanced abundance of $\alpha$ elements is characteristic of the original composition of a thick-disk low-mass star, which is consistent with a post-AGB interpretation. The observed large amplitude of the RV variations and a lack of a strong variability in $T_{\text {eff }}$ argue against pulsations. We therefore attributed the $\mathrm{RV}$ variations in $\mathrm{BD}+46^{\circ} 442$ to binarity (with a tentative detection of a companion spectrum in the CCF). We found orbital parameters of period 140.8 days, eccentricity 0.08 , and separation $<1$ AU. This adds to the several dozen post-AGB disk binaries with known orbital parameters. The orbital period falls on the short side of the period distribution of the other orbits.

Time-resolved spectroscopy has allowed to detect gas streams in $\mathrm{BD}+46^{\circ} 442$, indicating that it is an interactive binary. $\mathrm{H} \alpha$ (and to a lesser extent both higher Balmer lines and the $\mathrm{Na}$ I D) are found to alternate between double-peaked emission, which is characteristic of a Keplerian disk, and a P Cyg-like profile, which is characteristic of an outflow. The blue absorption reaches values up to $300 \mathrm{~km} \mathrm{~s}^{-1}$ and develops only around the giant's superior conjunction. We suggest that it is due to a jet that originates in the accretion disk around the companion. In addition, strong metal lines display central or slightly blue-shifted absorption within the photospheric profiles. Similar features are also observed in some massive interactive binaries and likely several other post-AGB disk sources (such as the famous Red Rectangle).

The discovery of mass transfer and, possibly, accretiondriven jets in post-AGB binaries opens a new avenue to exploring some of the long-standing puzzles of these objects, such as a variable mass-loss rate, a long lifetime of the circumbinary disks, a lack of extended dusty nebulae (Siódmiak et al. 2008; Lagadec et al. 2011), and the bipolar structure of the gaseous outflows (Bujarrabal et al. 2007). By uncovering more systems such as this in the course of our spectroscopic survey, aided by complimentary observations, we hope to pin-point the exact geometrical structures responsible for the various non-photospheric features. This will enable us to construct realistic hydrodynamical models, providing a more solid basis for the theory of binary evolution.

Acknowledgements. We would like to thank Dr. V. Kovtyukh for sharing his atomic data, Dr. P. Degroote for his SED tool, Dr. L. Winter for the use of her de-reddening script, the numerous observers who acquired our spectra, and the anonymous referee for useful suggestions. The HERMES project and team acknowledge support from the Fund for Scientific Research of Flanders, Belgium (FWO), support from the Research Council of K.U. Leuven (Belgium), support from the Fonds de la Recherche Scientifique, Belgium (FNRS), from the Royal Observatory of Belgium and from the Landessternwarte Tautenburg (Germany). This publication makes use of data products from AKARI, a JAXA project with the participation of ESA, and from the Wide-field Infrared Survey Explorer, which is a joint project of the University of California, Los Angeles, and the Jet Propulsion Laboratory/California Institute of Technology, funded by the National Aeronautics and Space Administration.

\section{References}

Ak, H., Chadima, P., Harmanec, P., et al. 2007, A\&A, 463, 233

Akashi, M., \& Soker, N. 2008, New A, 13, 157

Andersen, J., Nordstrom, B., Mayor, M., \& Polidan, R. S. 1988, A\&A, 207, 37

Arrieta, A., Torres-Peimbert, S., \& Georgiev, L. 2005, ApJ, 623, 252

Bagnulo, S., Jehin, E., Ledoux, C., et al. 2003, The Messenger, 114, 10

Bakker, E. J., Lambert, D. L., Van Winckel, H., et al. 1998, A\&A, 336, 263

Bergemann, M. 2011, MNRAS, 413, 2184

Bond, H. E. 1997, in Planetary Nebulae, ed. H. J. Habing, \& H. J. G. L. M. Lamers (Dordrecht: Kluwer Academic Publishers), IAU Symp., 180, 460

Budaj, J., \& Richards, M. T. 2004, Contributions of the Astronomical Observatory Skalnate Pleso, 34, 167

Bujarrabal, V., Van Winckel, H., Neri, R., et al. 2007, A\&A, 468, L45

Castelli, F., \& Kurucz, R. L. 2003, in Modelling of Stellar Atmospheres, ed. N. Piskunov, W. W. Weiss, \& D. F. Gray (San Francisco: ASP), IAU Symp., 210, 20P

Coelho, P., Barbuy, B., Meléndez, J., Schiavon, R. P., \& Castilho, B. V. 2005, A\&A, 443, 735

Cohen, M., Van Winckel, H., Bond, H. E., \& Gull, T. R. 2004, AJ, 127, 2362

De Marco, O. 2009, PASP, 121, 316

De Ruyter, S., Van Winckel, H., Maas, T., et al. 2006, A\&A, 448, 641

De Val-Borro, M., Karovska, M., \& Sasselov, D. 2009, ApJ, 700, 1148

Delbouille, L. et al. 1988, Photometric Atlas of the Solar Spectrum from 3000 to $10000 \AA$ A (Institut d'Astrophysique, Université de Liège)

Dermine, T., Izzard, R. G., Jorissen, A., \& Van Winckel, H. 2012, A\&A, submitted [arXiv: 1203.6471]

Deroo, P., Acke, B., Verhoelst, T., et al. 2007, A\&A, 474, L45

Fremat, Y., Houziaux, L., \& Andrillat, Y. 1996, MNRAS, 279, 25

Galazutdinov, G. A. 1992, Preprint SAO RAS, 92

Gielen, C., Van Winckel, H., Waters, L. B. F. M., Min, M., \& Dominik, C. 2007, A\&A, 475, 629

Gielen, C., Van Winckel, H., Min, M., Waters, L. B. F. M., \& Lloyd Evans, T. 2008, A\&A, 490, 725

Gielen, C., Van Winckel, H., Reyniers, M., et al. 2009, A\&A, 508, 1391

Gielen, C., Bouwman, J., Van Winckel, H., et al. 2011, A\&A, 533, A99

Ginestet, N., \& Carquillat, J. M. 2002, ApJS, 143, 513

Giridhar, S., Lambert, D. L., Reddy, B. E., Gonzalez, G., \& Yong, D. 2005, ApJ, 627,432

Gorlova, N., et al. 2012, in Asiago meeting on symbiotic stars, ed. U. Siviero, \& A. Munari, Baltic Astr. special issue, in press

Grevesse, N., Noels, A., \& Sauval, A. J. 1996, in Cosmic Abundances, ed. S. S. Holt, \& G. Sonneborn (San Francisco: ASP), ASP Conf. Ser., 99, 117

Grundstrom, E. D., Gies, D. R., Hillwig, T. C., et al. 2007, ApJ, 667, 505

Harmanec, P. 2002, Astron. Nachr., 323, 87

Hinkle, K. H., Brittain, S. D., \& Lambert, D. L. 2007, ApJ, 664, 501

Ishihara, D., Onaka, T., Kataza, H., et al. 2010, A\&A, 514, A1

Jorissen, A. 1999, in Asymptotic Giant Branch Stars, ed. T. Le Bertre, A. Lebre, \& C. Waelkens (San Francisco: ASP), IAU Symp., 191, 437

Kameswara Rao, N., Goswami, A., \& Lambert, D. L. 2002, MNRAS, 334, 129 
Kovtyukh, V. V., \& Andrievsky, S. M. 1999, A\&A, 351, 597

Kurucz, R. L. 1992, in The Stellar Populations of Galaxies, ed. B. Barbuy, \&

A. Renzini (Dordrecht: Kluwer Academic Publishers), IAU Symp., 149, 225

Kurucz, R. L., Furenlid, I., Brault, J., \& Testerman, L. 1984, The Solar Flux

Atlas from 296 to $1300 \mathrm{~nm}$, National Solar Observatory (New Mexico: Aura)

Lagadec, E., Verhoelst, T., Mékarnia, D., et al. 2011, MNRAS, 417, 32

Livio, M., \& Soker, N. 1988, ApJ, 329, 764

Lobel, A. 2008, J. Phys. Conf. Ser., 130, 2015

Lobel, A. 2011, Can. J. Phys., 89, 395

Lubow, S. H., \& Artymowicz, P. 1996, in Evolutionary Processes in Binary Stars, ed. R. A. M. J. Wijers, M. B. Davies, \& C. A. Tout (Dordrecht: Kluwer Academic Publishers), NATO ASIC Proc., 477, 53

Maas, T., Van Winckel, H., \& Waelkens, C. 2002, A\&A, 386, 504

Maas, T., Van Winckel, H., Lloyd Evans, T., et al. 2003, A\&A, 405, 271

Maas, T., Van Winckel, H., \& Lloyd Evans, T. 2005, A\&A, 429, 297

Mashonkina, L., Gehren, T., Shi, J.-R., Korn, A. J., \& Grupp, F. 2011, A\&A, 528, A87

Mastrodemos, N., \& Morris, M. 1999, ApJ, 523, 357

Mennickent, R. E., \& Smith, M. A. 2010, MNRAS, 407, 734

Miller, B., Budaj, J., Richards, M., Koubský, P., \& Peters, G. J. 2007, ApJ, 656, 1075

Min, M., Dullemond, C. P., Dominik, C., de Koter, A., \& Hovenier, J. W. 2009, A\&A, 497, 155

Mohamed, S., \& Podsiadlowski, P. 2011, in Asymmetric Planetary Nebulae 5 Conference (Manchester, UK: Jodrell Bank Centre for Astrophysics), 295

Mora, A., Eiroa, C., Natta, A., et al. 2004, A\&A, 419, 225

Munari, U., \& Castelli, F. 2000, A\&AS, 141, 141

Mürset, U., Dumm, T., Isenegger, S., et al. 2000, A\&A, 353, 952

Nariai, K. 1967, PASJ, 19, 564

Netolický, M., Bonneau, D., Chesneau, O., et al. 2009, A\&A, 499, 827

Paczyński, B. 1971, ARA\&A, 9, 183

Peters, G. J. 1989, Space Sci. Rev., 50, 9

Plavec, M. J. 1988, AJ, 96, 755

Pollard, K. R., Cottrell, P. L., Lawson, W. A., Albrow, M. D., \& Tobin, W. 1997, MNRAS, 286, 1

Przybilla, N., \& Butler, K. 2001, A\&A, 379, 955

Quiroga, C., Mikołajewska, J., Brandi, E., Ferrer, O., \& García, L. 2002, A\&A, 387,139

Raskin, G., Van Winckel, H., Hensberge, H., et al. 2011, A\&A, 526, A69

Reddy, B. E., Lambert, D. L., \& Allende Prieto, C. 2006, MNRAS, 367, 1329

Reid, M. J., Menten, K. M., Zheng, X. W., et al. 2009, ApJ, 700, 137

Rentzsch-Holm, I. 1996, A\&A, 312, 966

Richards, M. T., \& Albright, G. E. 1999, ApJS, 123, 537
Robinson, K., Bode, M. F., Skopal, A., Ivison, R. J., \& Meaburn, J. 1994, MNRAS, 269, 1

Sahai, R., Brillant, S., Livio, M., et al. 2002, ApJ, 573, L123

Sánchez Contreras, C., Sahai, R., Gil de Paz, A., \& Goodrich, R. 2008, ApJS, 179,166

Sandquist, E. L., Taam, R. E., Chen, X., Bodenheimer, P., \& Burkert, A. 1998 ApJ, 500, 909

Siódmiak, N., Meixner, M., Ueta, T., et al. 2008, ApJ, 677, 382

Skopal, A. 2006, A\&A, 457, 1003

Skopal, A., Pribulla, T., Budaj, J., et al. 2009, ApJ, 690, 1222

Slettebak, A. 1986, PASP, 98, 867

Sudar, D., Harmanec, P., Lehmann, H., et al. 2011, A\&A, 528, A146

Sumangala Rao, S., Giridhar, S., \& Lambert, D. L. 2012, MNRAS, 419, 1254

Szczerba, R., Siódmiak, N., Stasińska, G., \& Borkowski, J. 2007, A\&A, 469, 799

Takeda, Y. 1992, PASJ, 44, 649

Takeda, Y., Taguchi, H., Yoshioka, K., et al. 2007, PASJ, 59, 1127

Thomas, J. D., Witt, A. N., Aufdenberg, J. P., et al. 2011, MNRAS, 417, 2860

Usenko, I. A., Miroshnichenko, A. S., Klochkova, V. G., \& Yushkin, M. V. 2005, MNRAS, 362, 1219

Van Aarle, E., Van Winckel, H., Lloyd Evans, T., et al. 2011, A\&A, 530, A90

Van Eck, S., \& Jorissen, A. 2002, A\&A, 396, 599

Van Winckel, H. 2003, ARA\&A, 41, 391

Van Winckel, H., Duerbeck, H. W., \& Schwarz, H. E. 1993, A\&AS, 102, 401

Van Winckel, H., Waelkens, C., \& Waters, L. B. F. M. 1995, A\&A, 293, L25

Van Winckel, H., Lloyd Evans, T., Briquet, M., et al. 2009, A\&A, 505, 1221

Van Winckel, H., Jorissen, A., Gorlova, N., et al. 2010, Mem. Soc. Astron. It., 81,1022

Velázquez, P. F., Steffen, W., Raga, A. C., et al. 2011, ApJ, 734, 57

Waelkens, C., Lamers, H. J. G. L. M., Waters, L. B. F. M., et al. 1991, A\&A 242,433

Waelkens, C., Van Winckel, H., Waters, L. B. F. M., \& Bakker, E. J. 1996, A\&A, 314, L17

Waters, L. B. F. M., Trams, N. R., \& Waelkens, C. 1992, A\&A, 262, L37

Waters, L. B. F. M., Waelkens, C., Mayor, M., \& Trams, N. R. 1993a, A\&A, 269,242

Waters, L. B. F. M., Waelkens, C., \& Trams, N. R. 1993b, in ESO Conf. and Workshop Proc., Second ESO/CTIO Workshop on Mass loss on the AGB and beyond, ed. H. E. Schwarz (Garching: ESO), 46, 298

Weiland, J. L., Shore, S. N., Beaver, E. A., Lyons, R. W., \& Rosenblatt, E. I. 1995, ApJ, 447, 401

Witt, A. N., Vijh, U. P., Hobbs, L. M., et al. 2009, ApJ, 693, 1946

Wright, E. L., Eisenhardt, P. R. M., Mainzer, A. K., et al. 2010, AJ, 140, 1868

Pages 14 to 16 are available in the electronic edition of the journal at http://www . aanda. org 


\section{Appendix A: Line profiles and radial velocities}

Figure A.1 shows $\mathrm{H} \alpha$ and three other Balmer lines in $\mathrm{BD}+46^{\circ} 442$ ordered according to the orbital phase, where each of the five orbital cycles (covering the first 1.5 years of observations) is designated with a different colour. A correlation with the orbital phase is obvious. The central absorption in $\mathrm{H} \alpha$ is not always aligned with the photospheric velocity, which means that the profile cannot be represented by a simple superposition of a broad emission line with a photospheric absorption line. Close to the giant's inferior conjunction $(\phi=0.75), \mathrm{H} \alpha$ exhibits a doublepeak emission line, while near superior conjunction $(\phi=0.25)$ the blue peak is replaced by an extended blue absorption, as can also be seen in the higher Balmer lines.

Figure A.2 shows the behaviour of some metal lines and the $\mathrm{CCF}$, which represents the behaviour of the majority of the photospheric lines that are symmetric and move with the orbital velocity. Low-excitation $\left(\chi_{\text {low }}=0-3 \mathrm{eV}\right)$, strong $(E W>300 \mathrm{~m} \AA)$ lines, on the other hand, show additional circumstellar and interstellar (in Na I D) components, particularly for phases in the range $\phi=0.9-1.1$.
Table A.1. Radial velocities of $\mathrm{BD}+46^{\circ} 442$.

\begin{tabular}{|c|c|c|c|}
\hline $\bar{N}$ & $\begin{array}{c}\text { JD } 2455000+ \\
\text { (d) }\end{array}$ & $\begin{array}{c}\mathrm{RV} \\
\left(\mathrm{km} \mathrm{s}^{-1}\right)\end{array}$ & $\begin{array}{c}\sigma \\
\left(\mathrm{km} \mathrm{s}^{-1}\right)\end{array}$ \\
\hline 1 & 31.71 & $\begin{array}{l}-89.62 \\
\end{array}$ & 0.26 \\
\hline 2 & 42.70 & -80.72 & 0.30 \\
\hline 3 & 60.70 & -75.28 & 0.33 \\
\hline 4 & 60.72 & -75.29 & 0.33 \\
\hline 5 & 85.64 & -92.73 & 0.27 \\
\hline 6 & 92.64 & -100.15 & 0.28 \\
\hline 7 & 100.62 & -109.71 & 0.28 \\
\hline 8 & 125.49 & -122.42 & 0.32 \\
\hline 9 & 131.53 & -120.76 & 0.30 \\
\hline 10 & 156.40 & -102.56 & 0.26 \\
\hline 11 & 159.55 & -100.60 & 0.26 \\
\hline 12 & 159.56 & -100.42 & 0.27 \\
\hline 13 & 199.33 & -75.91 & 0.30 \\
\hline 14 & 216.38 & -83.05 & 0.29 \\
\hline 15 & 224.36 & -90.37 & 0.29 \\
\hline 16 & 421.63 & -116.87 & 0.30 \\
\hline 17 & 425.67 & -112.94 & 0.30 \\
\hline 18 & 432.59 & -106.89 & 0.27 \\
\hline 19 & 468.66 & -78.39 & 0.30 \\
\hline 20 & 471.62 & -76.86 & 0.32 \\
\hline 21 & 499.54 & -83.24 & 0.26 \\
\hline 22 & 507.47 & -91.20 & 0.27 \\
\hline 23 & 553.48 & -123.07 & 0.27 \\
\hline 24 & 573.34 & -107.14 & 0.25 \\
\hline 25 & 581.34 & -100.30 & 0.22 \\
\hline 26 & 767.71 & -76.56 & 0.30 \\
\hline 27 & 769.71 & -77.36 & 0.30 \\
\hline 28 & 776.73 & -80.47 & 0.29 \\
\hline 29 & 783.69 & -85.43 & 0.27 \\
\hline 30 & 791.74 & -94.47 & 0.25 \\
\hline 31 & 797.73 & -101.45 & 0.26 \\
\hline 32 & 826.65 & -123.22 & 0.28 \\
\hline 33 & 835.57 & -121.05 & 0.29 \\
\hline 34 & 841.50 & -118.54 & 0.28 \\
\hline 35 & 842.59 & -117.29 & 0.30 \\
\hline 36 & 845.61 & -114.46 & 0.29 \\
\hline 37 & 848.41 & -112.60 & 0.28 \\
\hline 38 & 852.52 & -109.55 & 0.25 \\
\hline 39 & 857.64 & -104.90 & 0.26 \\
\hline 40 & 860.60 & -102.75 & 0.25 \\
\hline 41 & 866.54 & -98.31 & 0.25 \\
\hline 42 & 870.56 & -95.45 & 0.25 \\
\hline 43 & 872.61 & -92.87 & 0.24 \\
\hline 44 & 880.56 & -84.86 & 0.26 \\
\hline 45 & 882.46 & -83.74 & 0.26 \\
\hline 46 & 884.55 & -82.57 & 0.27 \\
\hline 47 & 886.49 & -81.61 & 0.26 \\
\hline 48 & 888.55 & -80.62 & 0.25 \\
\hline 49 & 890.52 & -79.87 & 0.25 \\
\hline 50 & 890.54 & -79.79 & 0.25 \\
\hline 51 & 903.38 & -74.61 & 0.25 \\
\hline 52 & 911.41 & -77.25 & 0.28 \\
\hline 53 & 934.41 & -96.74 & 0.30 \\
\hline 54 & 940.36 & -105.17 & 0.31 \\
\hline 55 & 943.44 & -108.88 & 0.30 \\
\hline 56 & 948.40 & -114.77 & 0.28 \\
\hline 57 & 953.36 & -118.43 & 0.27 \\
\hline 58 & 956.40 & -119.74 & 0.26 \\
\hline 59 & 957.48 & -120.08 & 0.26 \\
\hline 60 & 968.45 & -121.61 & 0.33 \\
\hline
\end{tabular}

Notes. Radial velocities (barycentric correction included) measured with HERMES over the period July 2009-February 2012. 
N. Gorlova et al.: A newly discovered evolved binary with a disk

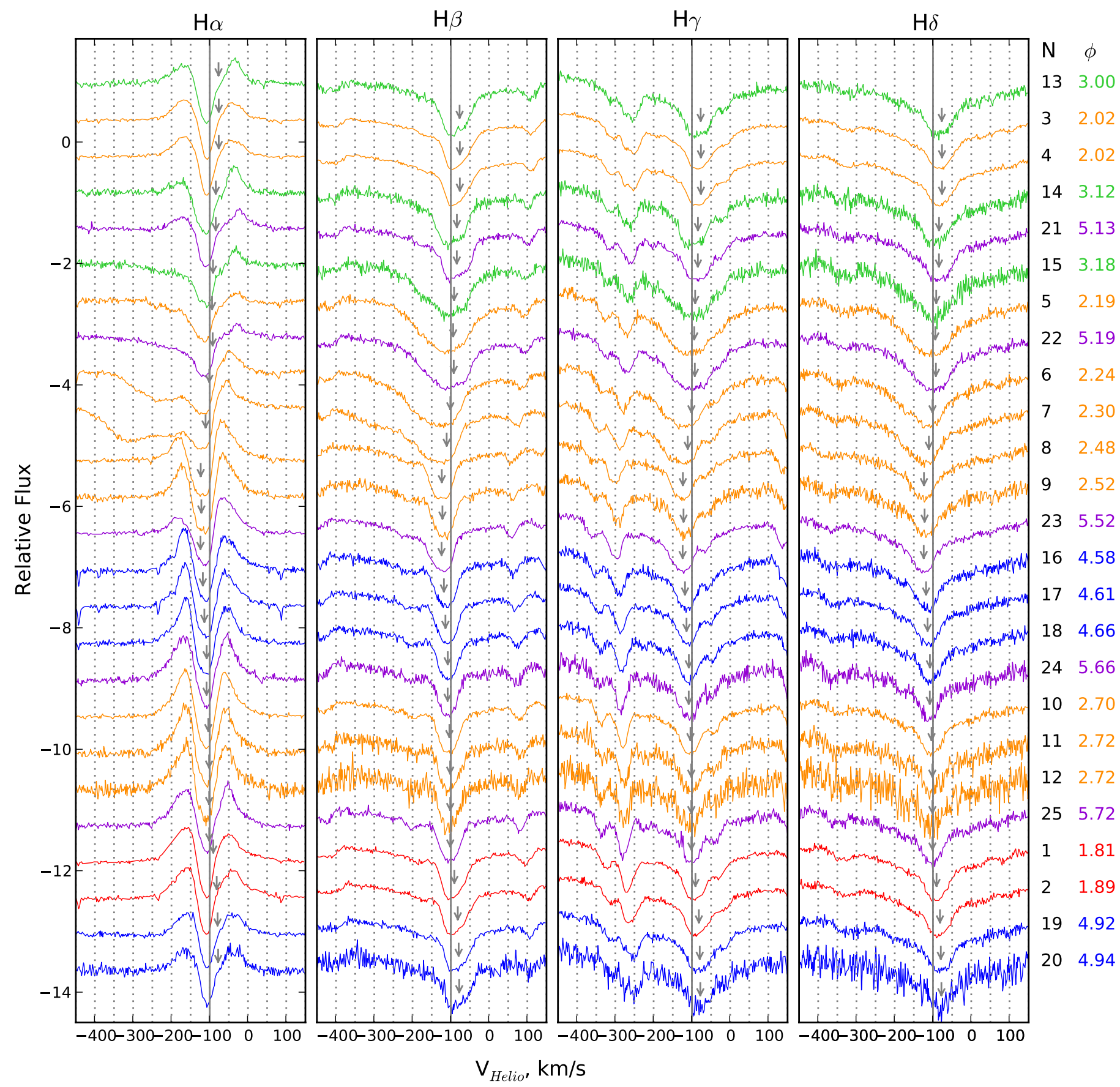

Fig. A.1. Balmer lines as a function of the RV phase $(\phi=0$ corresponds to the maximum redshift). Different colours denote different orbital cycles. A solid vertical line marks our systemic velocity of $-98.9 \mathrm{~km} \mathrm{~s}^{-1}$, while dotted lines mark $50 \mathrm{~km} \mathrm{~s}^{-1}$ intervals from it. The arrows mark the photospheric velocity according to the CCF. 

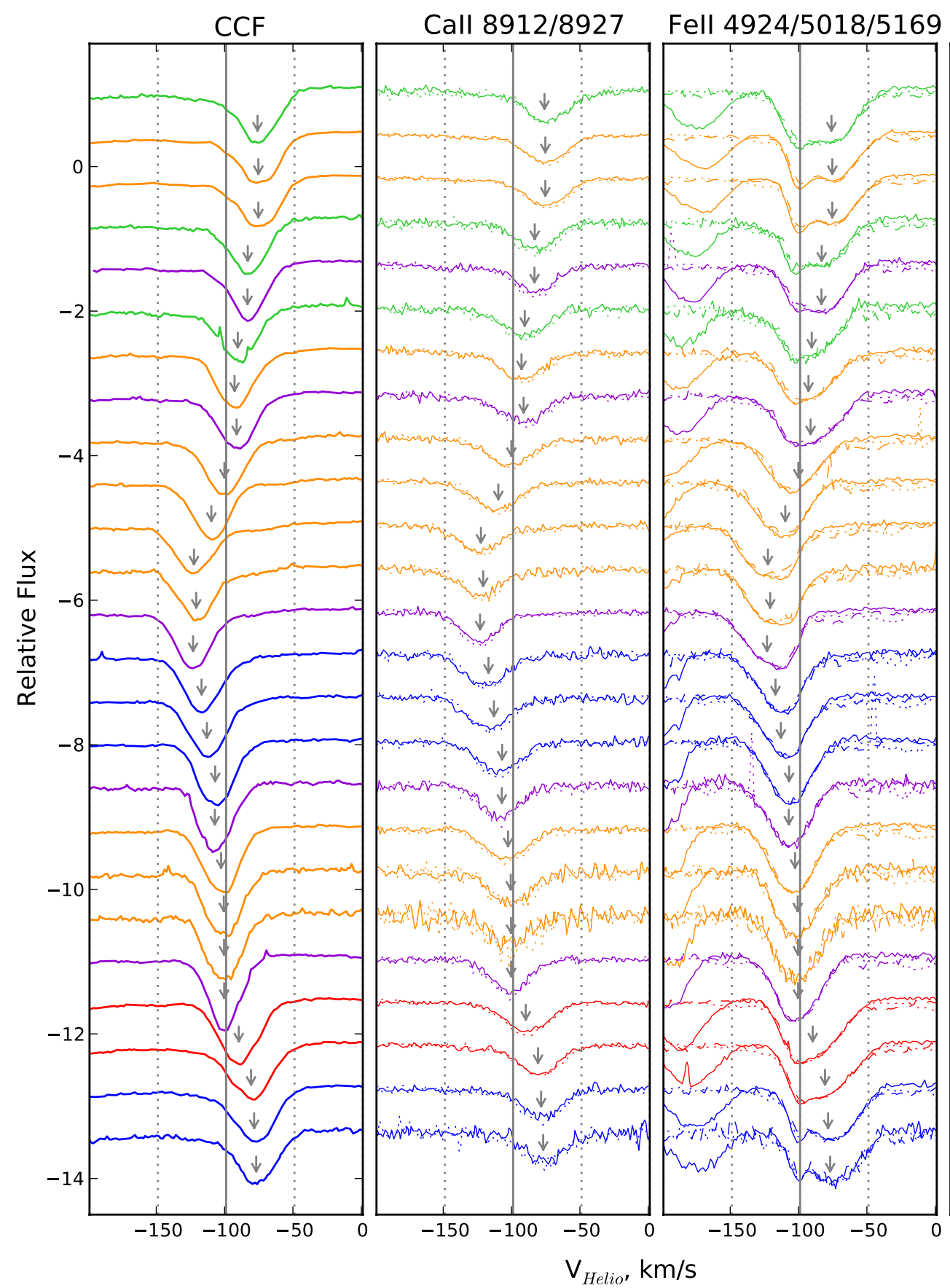

Nal 5890/5896

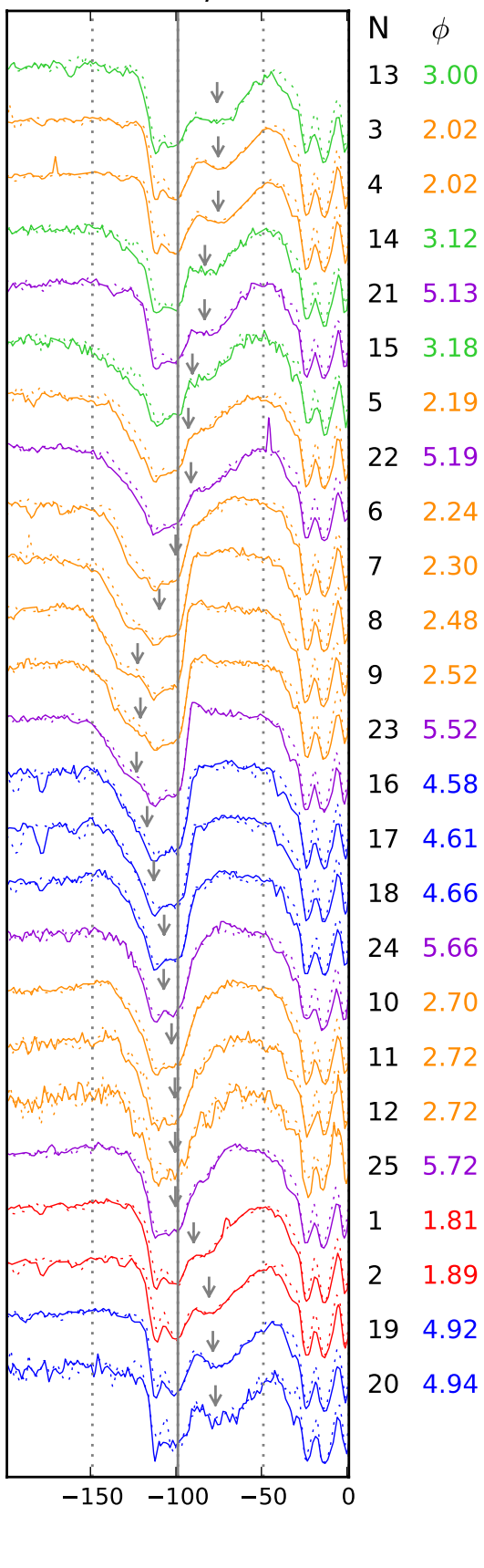

Fig. A.2. Same as in Fig. A.1, only for the CCF and the representative metal lines of different strengths: Ca II 4d-4f doublet, Fe II (42) triplet, and the Na I D doublet. In each panel, different line styles (solid, dashed, dotted) designate different members of the same multiplet. 